\title{
Mathematical Model and Analysis of the Water-Lubricated Hydrostatic Journal Bearings considering the Translational and Tilting Motions
}

\author{
Hui-Hui Feng, ${ }^{1}$ Chun-Dong Xu, ${ }^{1}$ and Jie Wan ${ }^{2}$ \\ ${ }^{1}$ School of Mechanical Engineering, Southeast University, Nanjing 211189, China \\ ${ }^{2}$ CSR Qishuyan Institute Co., Ltd., Changzhou 213011, China \\ Correspondence should be addressed to Hui-Hui Feng; fhhwjmail@163.com
}

Received 14 April 2014; Revised 13 June 2014; Accepted 17 June 2014; Published 17 July 2014

Academic Editor: Nam-Il Kim

Copyright (C) 2014 Hui-Hui Feng et al. This is an open access article distributed under the Creative Commons Attribution License, which permits unrestricted use, distribution, and reproduction in any medium, provided the original work is properly cited.

\begin{abstract}
The water-lubricated bearings have been paid attention for their advantages to reduce the power loss and temperature rise and increase load capacity at high speed. To fully study the complete dynamic coefficients of two water-lubricated, hydrostatic journal bearings used to support a rigid rotor, a four-degree-of-freedom model considering the translational and tilting motion is presented. The effects of tilting ratio, rotary speed, and eccentricity ratio on the static and dynamic performances of the bearings are investigated. The bulk turbulent Reynolds equation is adopted. The finite difference method and a linear perturbation method are used to calculate the zeroth- and first-order pressure fields to obtain the static and dynamic coefficients. The results suggest that when the tilting ratio is smaller than 0.4 or the eccentricity ratio is smaller than 0.1 , the static and dynamic characteristics are relatively insensitive to the tilting and eccentricity ratios; however, for larger tilting or eccentricity ratios, the tilting and eccentric effects should be fully considered. Meanwhile, the rotary speed significantly affects the performance of the hydrostatic, waterlubricated bearings.
\end{abstract}

\section{Introduction}

Hydrostatic journal bearings are applied widely in spindlebearing systems owning to their favorable performance characteristics. However, with the requirement of higher machining speed, the limitations of the conventional oil film bearings are apparently due to their remarkable power loss as well as the temperature rise. Therefore, the water-lubricated bearings were developed and have been studied to fulfill the targets of lower power loss, lower temperature rise, and heavier load capacity at high speed.

Many studies related to water-lubricated bearings have been reported in the literatures in the past few years. Liu et al. compared the oil-lubricated and water-lubricated hybrid sliding bearings, and the results show that the latter benefits more from improved processing precision and efficiency [1]. Yuan et al. study the static and dynamic characteristics of waterlubricated hybrid journal bearings compensated by short capillaries [2]. Yoshimoto et al. investigated the static characteristics of water-lubricated hydrostatic conical bearings with spiral grooves for high speed spindles [3]. Gao et al. analyzed the effects of eccentricity ration on pressure distribution of water-lubricated plain journal bearings by computational fluid dynamics (CFD) [4]. In summary, extensive researches have been conducted in the area of water lubricated bearings in various aspects: numerical methods [1]; performance of the bearings with various geometries $[2,3,5,6]$; effects of various kinds of restrictors upon performance of a bearing [7], et al. However, their studies were restricted to the static or dynamic characteristics of plain or grooved journal bearings considering only translational motion of the journal.

In actual practice, the bearings and the journals may not be properly aligned as a result of improper assembly or noncentral loading. As a consequence, not only should the translational motion of the rotor be studied, but also the tilting motion of the rotor should be investigated. 


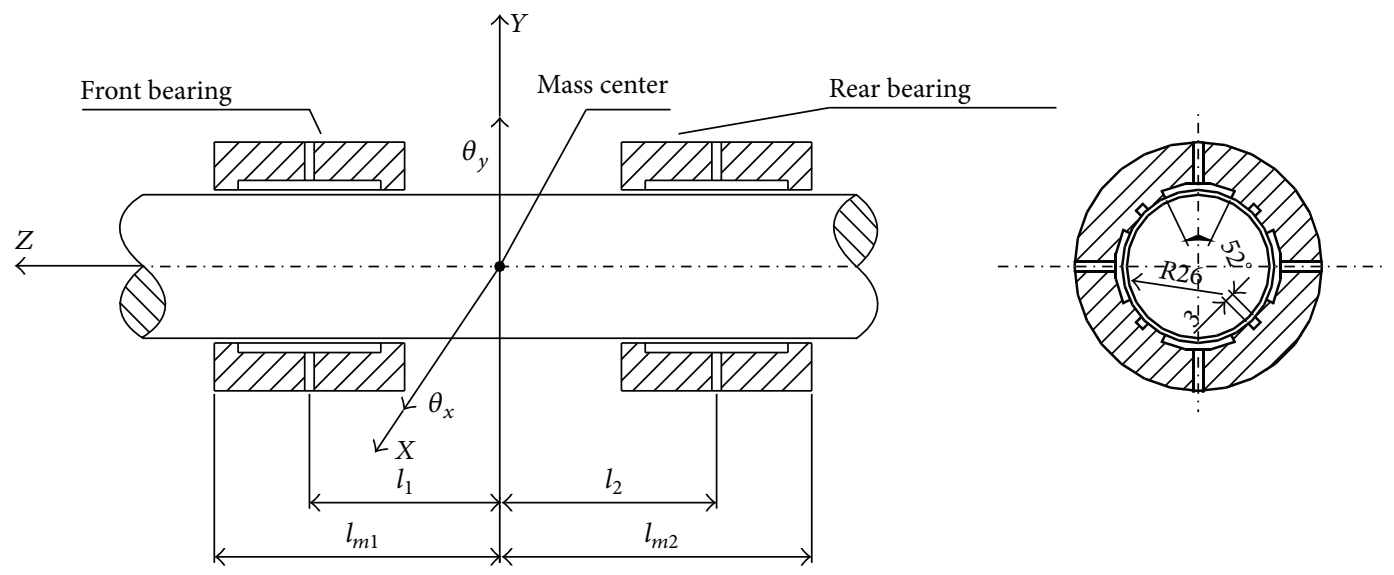

FIGURE 1: The arrangement and coordinate system of the bearings [6].

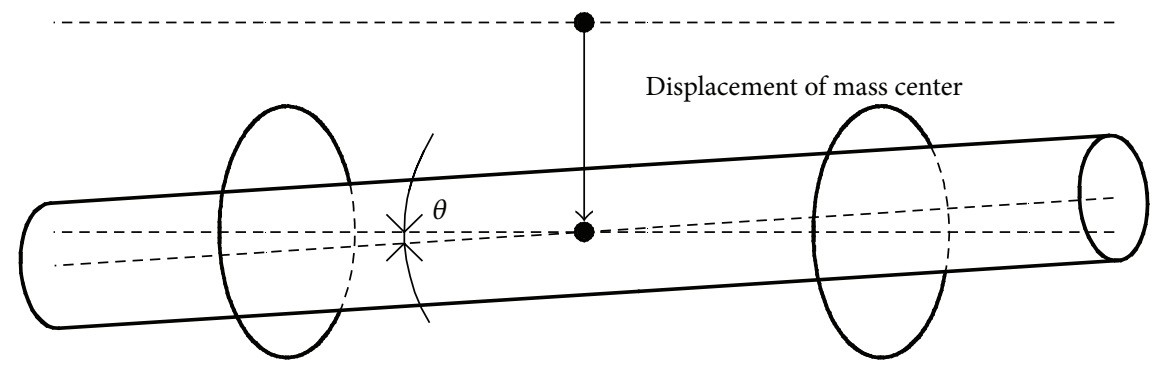

FIGURE 2: The rigid rotor-bearing model.

As a result, the complete stiffness and damping coefficients of a journal bearing, which is important to the vibration of a rotor, should be taken into consideration in four degrees of freedom, including the translation in $x, y$ direction and tilting about the $x$ - and $y$-axis. Numerous studies concerning the tilting motion of journal are available in the literatures [8-10]. Recently, Jang et al. [11-13] thoroughly investigated the dynamic characteristics of the journal and groove thrust bearings used to support a HDD spindle considering both of the translational motion and tilting motion of the journal. Results show that the tilting motions have an important role in the dynamic characteristics of the proposed bearings.

Unlike the conventional oil film bearings, waterlubricated bearings utilized in spindle are different in working conditions and characteristics. However, for hydrostatic water-lubricated journal bearings, we are not aware of any previous investigations to study their dynamic characteristics considering the translational and titling motions. Therefore, in this work, we aim to fully study the complete dynamic coefficients for two water-lubricated, hydrostatic journal bearings used to support a rigid rotor. The dynamic characteristics will be categorized into four groups: coefficients of force to displacement, coefficients of force to angle, coefficients of moment to angle, and coefficients of moment to displacement. In the present study, in order to fully study the variations of the complete static and dynamic characteristics of the proposed water-lubricated bearings, the influences of the tilting ratio, rotary speed, and eccentricity ratio on the bearings have been studied.

\section{Mathematical Models}

Figure 1 shows the schematic representations for a rigid rotor supported by a pair of identical water-lubricated journal bearings, as well as the geometry of a hydrostatic, waterlubricated journal bearing. Pressed water enters the bearing across an orifice restrictor, flows into the film lands, and then exits the bearing. The recess pressure is regarded as uniform. As shown in Figure 1, the rigid rotor moved in an inertial reference frame $C-X Y Z$; the rotor tilts about $X$ by an angle $\theta_{x}$ and tilts about $Y$ by an angle $\theta_{y}$. The rigid rotor-bearing model is shown in Figure 2.

2.1. Reynolds Equation. For an isoviscous, incompressible fluid, the Reynolds equation governing the turbulent bulk flow in nondimensional form is given as

$$
\frac{\partial}{\partial \varphi}\left(\frac{G_{x} H^{3}}{\mu} \frac{\partial P}{\partial \varphi}\right)+\frac{r^{2}}{l^{2}} \frac{\partial}{\partial \lambda}\left(\frac{G_{z} H^{3}}{\mu} \frac{\partial P}{\partial \lambda}\right)=\Gamma_{1} \frac{\partial H}{\partial \varphi}+\Gamma_{2} \frac{\partial H}{\partial \tau}
$$


where $\Gamma_{1}=\mu_{0} \Omega / 2 P_{s} \vartheta^{2}, \Gamma_{2}=\mu_{0} \Omega / p_{s} \vartheta^{2}, \vartheta=c / r$, and the details of the turbulent coefficients $G_{x}, G_{z}$ can be obtained as follows $[6,14-16]$ :

$$
\begin{gathered}
V_{x}=-G_{x} \frac{h^{2}}{\mu} \frac{\partial p}{\partial x}+G_{J} \frac{U}{2}, \\
V_{Z}=-G_{Z} \frac{h^{2}}{\mu} \frac{\partial p}{\partial z}, \\
R_{B}=\frac{\rho h}{\mu}\left[V_{x}^{2}+V_{Z}^{2}\right]^{1 / 2}, \\
R_{J}=\frac{\rho h}{\mu}\left[\left(V_{x}-U\right)^{2}+V_{Z}^{2}\right]^{1 / 2}, \\
f_{J}=0.066 R_{J}^{-0.25}, \\
f_{B}=0.066 R_{B}^{-0.25}, \\
k_{J}=f_{J} R_{J}, \\
k_{B}=f_{B} R_{B}, \\
G_{x}=G_{z}=\frac{2}{\left(k_{J}+k_{B}\right)} .
\end{gathered}
$$

With the increase of rotary speed or film depth, the flow is likely to become turbulent from laminar state. The turbulent coefficients $G_{x}, G_{y}$, and $G_{z}$ dependent on the fluid velocity field are obtained as follows [15]:

$$
\begin{aligned}
& G_{x}=\min \left\{\frac{1}{12}, G_{x}\right\} \\
& G_{y}=\min \left\{\frac{1}{12}, G_{y}\right\} \\
& G_{z}=\min \left\{\frac{1}{12}, G_{z}\right\} .
\end{aligned}
$$

At the bearing exit plane, the pressure takes a constant value equal to the ambient pressure.

2.2. Continuity Equation. The dimensionless continuity equation at the recess is defined by the global balance between the flow through the orifice restrictor and the recess outflow into the film lands:

$$
\begin{aligned}
\lambda \sqrt{\left(1-P_{r}\right)}= & \int_{S_{1}+S_{3}}\left(\frac{6 \mu_{0} L r \Omega}{P_{s} c^{2}} H-\frac{12 L}{r} \frac{G_{x} H^{3}}{\bar{\mu}} \frac{\partial P}{\partial \varphi}\right) d \lambda \\
& +\int_{S_{2}+S_{4}} \frac{12 r}{L} \frac{G_{z} H^{3}}{\bar{\mu}} \frac{\partial P}{\partial \lambda} d \varphi,
\end{aligned}
$$

where, $\lambda=3 \sqrt{2} \pi \alpha d_{0}^{2} \mu /\left(c^{3} \sqrt{\rho P_{s}}\right), P_{r}$ is the recess pressure, $S_{1,3}$ is the circumferential recess boundary, and $S_{2,4}$ is the axial recess boundary.

2.3. Perturbation Analysis. The journal center rotates about its steady-state position $\left(x_{0}, y_{0}, \theta_{x 0}, \theta_{y 0}\right)$ with a small whirl which is generated from the variations due to the translations of the rotor mass center and variations due to the tilting angles [11-13]. For small amplitude motions, the dimensionless film thickness and pressure fields are expressed as the sum of a zeroth-order field and first-order field, describing the steadystate condition and perturbed motion, respectively.

The dimensionless perturbed film expression considering the tilting angles is [12]

$$
\begin{gathered}
H_{i, j}=H_{0 i, j}+\Delta \varepsilon_{x} \cdot \sin \theta_{i, j}+\Delta \varepsilon_{y} \cdot \cos \theta_{i, j}+\varphi_{J} \Delta \theta_{x}+\psi_{J} \Delta \theta_{y} \\
H_{0 i, j}=1+\varepsilon_{0} \cos \varphi_{i, j}+\varphi_{J} \theta_{x}+\psi_{J} \theta_{y},
\end{gathered}
$$

where

$$
\begin{aligned}
\varphi_{J} & =\frac{\gamma l_{i, j} \cos \theta_{x} \cos \theta_{i, j}}{c} \\
\psi_{J} & =-\frac{\gamma l_{i, j} \cos \theta_{y} \sin \theta_{i, j}}{c},
\end{aligned}
$$

where $l_{i, j}$ is the distance between the grid node of each bearing and the mass center of the rotor supported on the bearings, $\gamma=1$ for the front journal bearing, and $\gamma=-1$ for the rear journal bearing.

The dimensionless perturbed pressure expression is

$$
\begin{aligned}
P= & P_{0}+\frac{\partial P}{\partial \Delta \varepsilon x} \Delta \varepsilon x+\frac{\partial P}{\partial \Delta \varepsilon y} \Delta \varepsilon y+\frac{\partial P}{\partial \Delta \theta_{x}} \Delta \theta_{x}+\frac{\partial P}{\partial \Delta \theta_{y}} \Delta \theta_{y} \\
& +\frac{\partial P}{\partial \Delta \varepsilon \dot{x}} \Delta \varepsilon \dot{x}+\frac{\partial P}{\partial \Delta \varepsilon \dot{y}} \Delta \varepsilon \dot{y}+\frac{\partial P}{\partial \Delta \dot{\theta}_{x}} \Delta \dot{\theta}_{x}+\frac{\partial P}{\partial \Delta \dot{\theta}_{y}} \Delta \dot{\theta}_{y} .
\end{aligned}
$$

Substitution of the perturbed equations (5)-(7) into the Reynolds equation yields the zeroth- and first-order expressions:

$$
\begin{array}{r}
\frac{\partial}{\partial \varphi}\left(\frac{G_{x} H^{3}}{\mu} \frac{\partial P_{\xi}}{\partial \varphi}\right)+\frac{r^{2}}{l^{2}}\left(\frac{G_{z} H^{3}}{\mu} \frac{\partial P_{\xi}}{\partial \lambda}\right)=F_{\xi}, \\
\left(\xi=0, x, y, \theta_{x}, \theta_{y}, \dot{x}, \dot{y}, \dot{\theta}_{x}, \dot{\theta}_{y}\right) \\
F_{0}=\Gamma_{1} \frac{\partial H_{0}}{\partial \varphi} \\
F_{\varepsilon x}=-\frac{\partial}{\partial \varphi}\left[\frac{G_{x} 3 H_{0}^{2}}{\bar{\mu}} \sin \theta \frac{\partial P_{0}}{\partial \varphi}\right] \\
-\frac{r^{2}}{l^{2}} \frac{\partial}{\partial \lambda}\left[\frac{G_{z} 3 H_{0}^{2}}{\bar{\mu}} \sin \theta \frac{\partial P_{0}}{\partial \lambda}\right] \\
+\Gamma_{1}\left(\sum \frac{\partial(\sin \theta)}{\partial \varphi}\right)+\Gamma_{2}\left(\sum \frac{\partial(\sin \theta)}{\partial \tau}\right) \\
F_{\varepsilon y}=-\frac{\partial}{\partial \varphi}\left[\frac{G_{x} 3 H_{0}^{2}}{\bar{\mu}} \cos \theta \frac{\partial P_{0}}{\partial \varphi}\right] \\
-\frac{r^{2}}{l^{2}} \frac{\partial}{\partial \lambda}\left[\frac{G_{z} 3 H_{0}^{2}}{\bar{\mu}} \cos \theta \frac{\partial P_{0}}{\partial \lambda}\right]
\end{array}
$$




$$
\begin{gathered}
+\Gamma_{1}\left(\sum \frac{\partial(\cos \theta)}{\partial \varphi}\right)+\Gamma_{2}\left(\sum \frac{\partial(\cos \theta)}{\partial \tau}\right) \\
F_{\theta x}=-\frac{\partial}{\partial \varphi}\left[\frac{G_{x} 3 H_{0}^{2}}{\bar{\mu}} \varphi_{J} \frac{\partial P_{0}}{\partial \varphi}\right]-\frac{r^{2}}{l^{2}} \frac{\partial}{\partial \lambda}\left[\frac{G_{z} 3 H_{0}^{2}}{\bar{\mu}} \varphi_{J} \frac{\partial P_{0}}{\partial \lambda}\right] \\
+\Gamma_{1}\left(\sum \frac{\partial\left(\varphi_{J}\right)}{\partial \varphi}\right)+\Gamma_{2}\left(\sum \frac{\partial\left(\varphi_{J}\right)}{\partial \tau}\right) \\
F_{\theta y}=-\frac{\partial}{\partial \varphi}\left[\frac{G_{x} 3 H_{0}^{2}}{\bar{\mu}} \psi_{J} \frac{\partial P_{0}}{\partial \varphi}\right]-\frac{r^{2}}{l^{2}} \frac{\partial}{\partial \lambda}\left[\frac{G_{z} 3 H_{0}^{2}}{\bar{\mu}} \psi_{J} \frac{\partial P_{0}}{\partial \lambda}\right] \\
+\Gamma_{1}\left(\sum \frac{\partial\left(\psi_{J}\right)}{\partial \varphi}\right)+\Gamma_{2}\left(\sum \frac{\partial\left(\psi_{J}\right)}{\partial \tau}\right)
\end{gathered}
$$

$$
\begin{gathered}
F_{\varepsilon \dot{x}}=\Gamma_{2} \sin \theta \\
F_{\varepsilon \dot{y}}=\Gamma_{2} \cos \theta \\
F_{\varepsilon \dot{\theta}_{x}}=\Gamma_{2} \varphi_{J} \\
F_{\varepsilon \dot{\theta}_{y}}=\Gamma_{2} \psi_{J} .
\end{gathered}
$$

The perturbed quality into the orifice diameter can be obtained by Taylor expansion:

$$
Q_{\text {in }}=\lambda\left(1-P_{r 0}\right)^{1 / 2}-\frac{\lambda}{2}\left(1-P_{r 0}\right)^{-1 / 2}\left(P_{r}-P_{r 0}\right) .
$$

Substitution of the perturbed equations (5)-(7) into the continuity equation yields the zeroth- and first-order expressions:

$$
\begin{aligned}
& \lambda \sqrt{\left(1-P_{r 0}\right)}=\int_{S_{1}+S_{3}}\left(\frac{6 \mu_{0} L r \Omega}{P_{s} c^{2}} H_{0 i, j}-\frac{12 L}{r} \frac{G_{x} H_{0 i, j}^{3}}{\bar{\mu}} \frac{\partial P_{0}}{\partial \varphi}\right) d \lambda \\
& +\int_{S_{2}+S_{4}} \frac{12 r}{L} \frac{G_{z} H_{0 i, j}^{3}}{\bar{\mu}} \frac{\partial P_{0}}{\partial \lambda} d \varphi, \\
& -\frac{\lambda}{2}\left(1-P_{r 0}\right)^{-1 / 2} P_{\xi}
\end{aligned}
$$

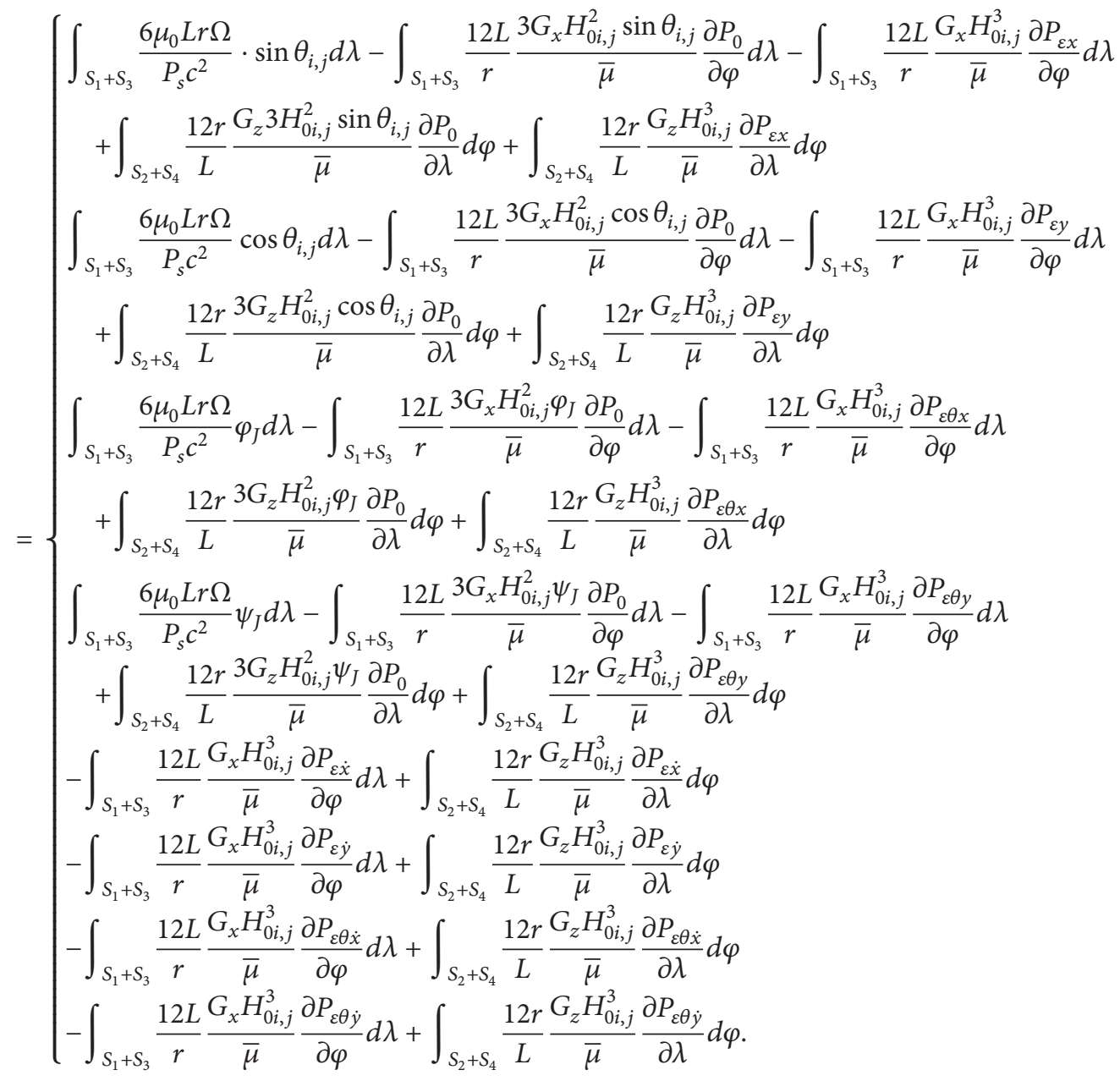


The finite difference method (FDM) and a successive over-relaxation (SOR) scheme are implemented to solve (1)(10) to find the pressure distribution. When the steady and perturbed pressure distributions are obtained, the static and dynamic coefficients can be solved.

2.4. Static Characteristics. The quality, frictional power loss, and pump power are calculated by integration of the pressure field on the bearing surfaces:

$$
\begin{gathered}
Q_{i n}=\lambda \sqrt{1-P_{r}}, \\
h_{f}=\frac{\mu(r \Omega)^{2}}{h_{0}} \iint r d \varphi d z+\frac{\mu \Omega^{2}}{h_{0}} \iint r^{3} d r d \varphi, \\
h_{p}=P_{s} Q .
\end{gathered}
$$

San Andres et al. carried out a systematic research on the water-lubricated hydrostatic journal bearings both theoretically and experimentally [17]. Numerical and experimental results show that predictions of the bearing performance characteristics like flow rate, load capacity, and rotor dynamic force coefficients are not affected by the small temperature variations $\left(\Delta T<10^{\circ} \mathrm{C}\right)$ in the water hydrostatic journal bearings. As a result, an adiabatic, isothermal assumption is made in this study. All the heat produced in the bearings by friction is considered absorbed by water film. The average elevated temperature is given by

$$
\Delta T=\frac{h_{f}+h_{p}}{\mathrm{Q} \rho C_{v}},
$$

where, $\rho$ is the density of water and $C_{v}$ is the specific heat capacity of water.

2.5. Dynamic Characteristics. The dynamic coefficients of the journal bearings can be calculated by integrating the perturbed pressure across the fluid film. There are 16 stiffness and damping coefficients, and the dynamic coefficients can be grouped into four categories: coefficients of force to displacement, coefficients of force to angle, coefficients of moment to angle, and coefficients of moment to displacement:

$$
\begin{aligned}
{\left[K_{J}\right] } & =\left[\begin{array}{cccc}
K_{x x} & K_{x y} & K_{x \theta_{x}} & K_{x \theta_{y}} \\
K_{y x} & K_{y y} & K_{y \theta_{x}} & K_{y \theta_{y}} \\
K_{M_{x} x} & K_{M_{x} y} & K_{M_{x} \theta_{x}} & K_{M_{x} \theta_{x}} \\
K_{M_{y} x} & K_{M_{y} y} & K_{M_{x} \theta_{x}} & K_{M_{x} \theta_{x}}
\end{array}\right]_{J} \\
& =-\iint\left[\begin{array}{c}
\sin \theta \\
\cos \theta \\
-\gamma l_{i, j} \cos \theta \\
\gamma l_{i, j} \sin \theta
\end{array}\right]\left[\begin{array}{llll}
P_{x} & P_{y} & P_{\theta_{x}} & P_{\theta_{y}}
\end{array}\right] d \varphi d \lambda,
\end{aligned}
$$

TABLE 1: Operating condition of the test bearing [17].

\begin{tabular}{lccc}
\hline Orifice diameter $(\mathrm{mm})$ & 2.49 & Supply temperature $\left({ }^{\circ} \mathrm{C}\right)$ & 55 \\
Length $(\mathrm{mm})$ & 76.2 & Supply pressure $(\mathrm{MPa})$ & 4 \\
Film thickness (um) & 127 & Rotary speed $(\mathrm{rpm})$ & 24600 \\
\hline
\end{tabular}

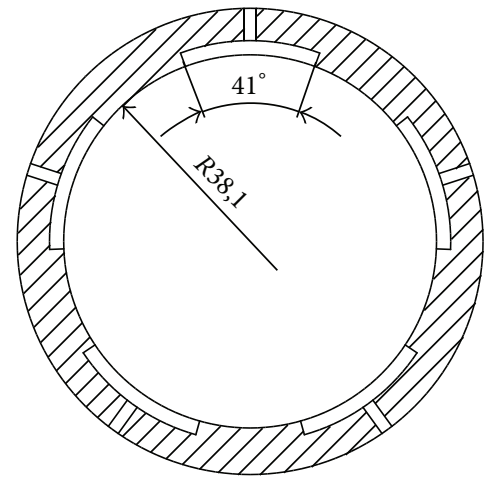

FIGURE 3: Geometry of the test water-lubricated hydrostatic journal bearing [17].

$$
\begin{aligned}
{\left[B_{J}\right] } & =\left[\begin{array}{cccc}
b_{x x} & b_{x y} & b_{x \theta_{x}} & b_{x \theta_{y}} \\
b_{y x} & b_{y y} & b_{y \theta_{x}} & b_{y \theta_{y}} \\
b_{M_{x} x} & b_{M_{x} y} & b_{M_{x} \theta_{x}} & b_{M_{x} \theta_{x}} \\
b_{M_{y} x} & b_{M_{y} y} & b_{M_{x} \theta_{x}} & b_{M_{x} \theta_{x}}
\end{array}\right]_{J} \\
& =-\iint\left[\begin{array}{c}
\sin \theta \\
\cos \theta \\
-\gamma l_{i, j} \cos \theta \\
\gamma l_{i, j} \sin \theta
\end{array}\right]\left[\begin{array}{llll}
P_{\dot{x}} & P_{\dot{y}} & P_{\dot{\theta}_{x}} & P_{\dot{\theta}_{y}}
\end{array}\right] d \varphi d \lambda .
\end{aligned}
$$

2.6. The Numerical Solution Procedure. The finite difference method (FDM) and a successive over-relaxation (SOR) scheme are implemented to solve (1)-(10) governing the flow in the film to find the pressure distribution. The overrelaxation factor always lies between (1 2). By trial and error, one can determine an optimum value of the relaxation factor for the fastest convergence. Normally, 1.7 is a good starting point for determining the relaxation factor [18]. In the numerical procedure implemented, any negative pressures calculated in the cavitation zone are arbitrarily set equal to zero (or ambient) pressure [18]. The pressure iterations are continued until the following convergence criterion is satisfied:

$$
\frac{\sum_{j=1}^{m} \sum_{i=1}^{n}\left|P_{i, j}^{(k)}-P_{i, j}^{(k-1)}\right|}{\sum_{j=1}^{m} \sum_{i=1}^{n}\left|P_{i, j}^{(k)}\right|} \leq \delta
$$

where $\delta$ is the convergence criteria, which is set as $10^{-3}$. Once the pressure field is established for the water film, other performance parameters follow from the pressure distribution. The steady and perturbed pressure distributions 


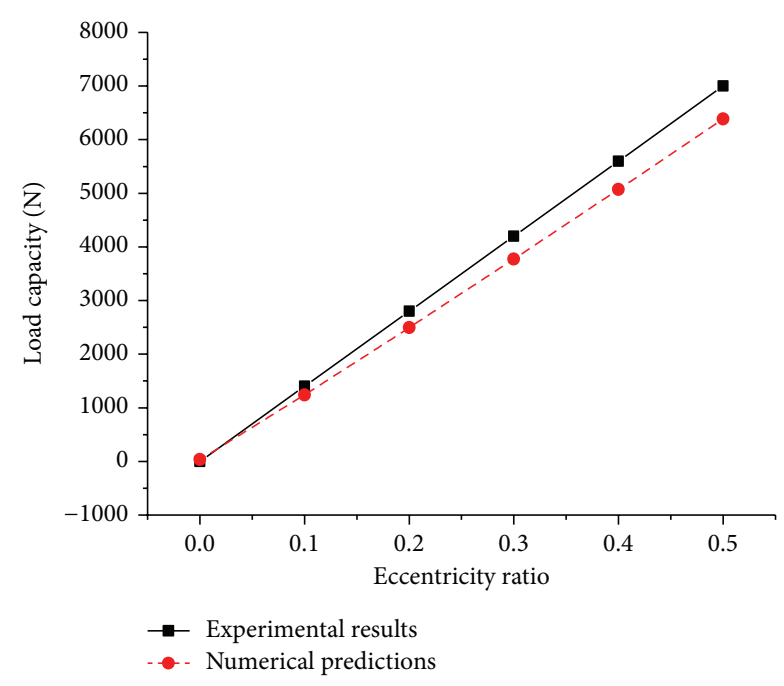

(a) Comparison of load capacity

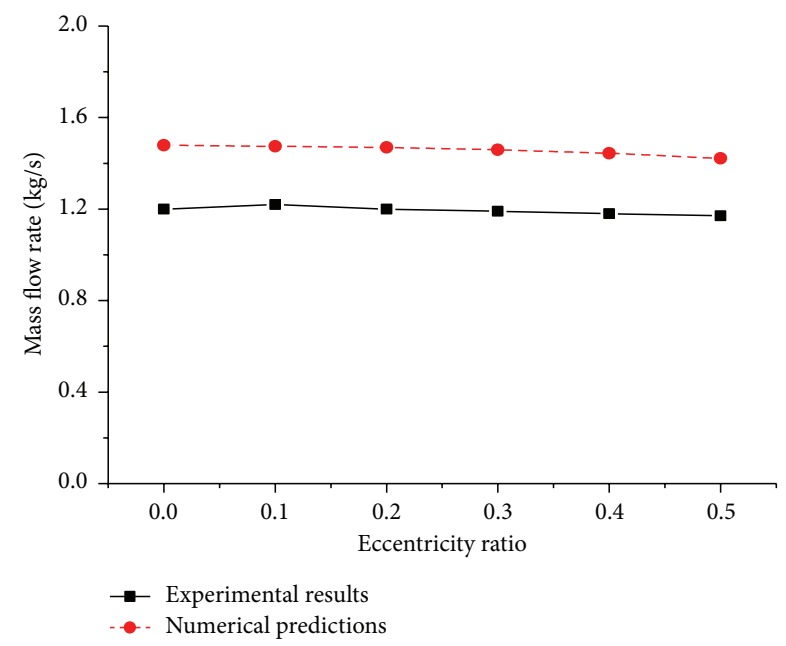

(b) Comparison of mass flow rate

FIGURE 4: Comparison of static performance of experimental results [17] and numerical predictions in respect to eccentricity ratio.

obtained are subsequently integrated to yield the desired static and dynamic coefficients.

The fluid film was discretized by rectangular grid with unequal intervals, which are $0.033,0.088$, and 0.1154 for the film land, recess, and return groove, respectively, in the circumferential direction and 0.02 and 0.04 for the film land and recess area in the axial direction. The total number of the grid is $31 \times 102$. It takes about 35 seconds for the static performance to achieve convergence. A few validation tests were made with a coarser grid of $31 \times 86$ and finer grid of $37 \times$ 158 with different intervals, and in no case did the predicted results of the static characteristics differ by more than 0.1 percent from those obtained by the initial grid.

\section{Results and Discussion}

3.1. Comparisons of Present Solution with Experimental Results. The present numerical solution has been correlated

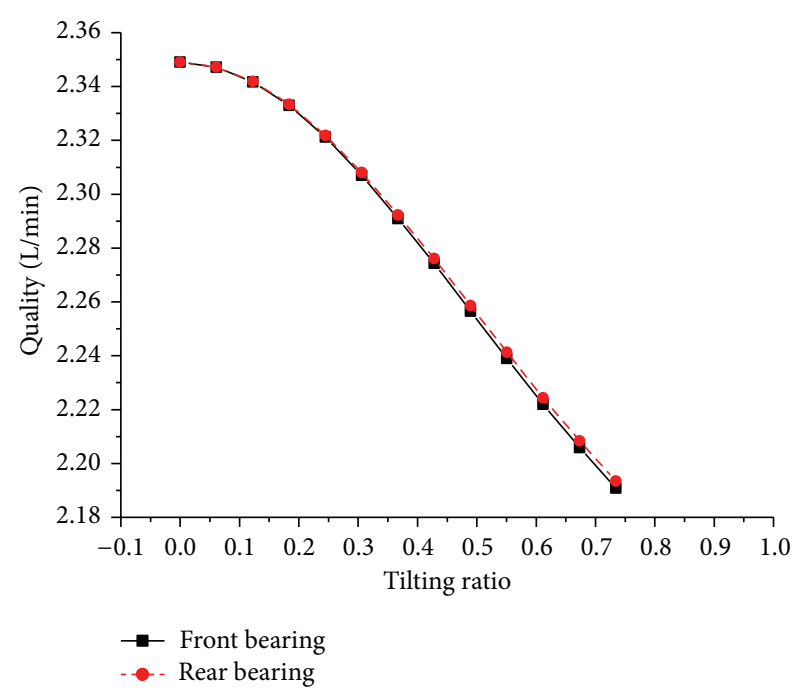

(a) The effect of tilting angle on the quality

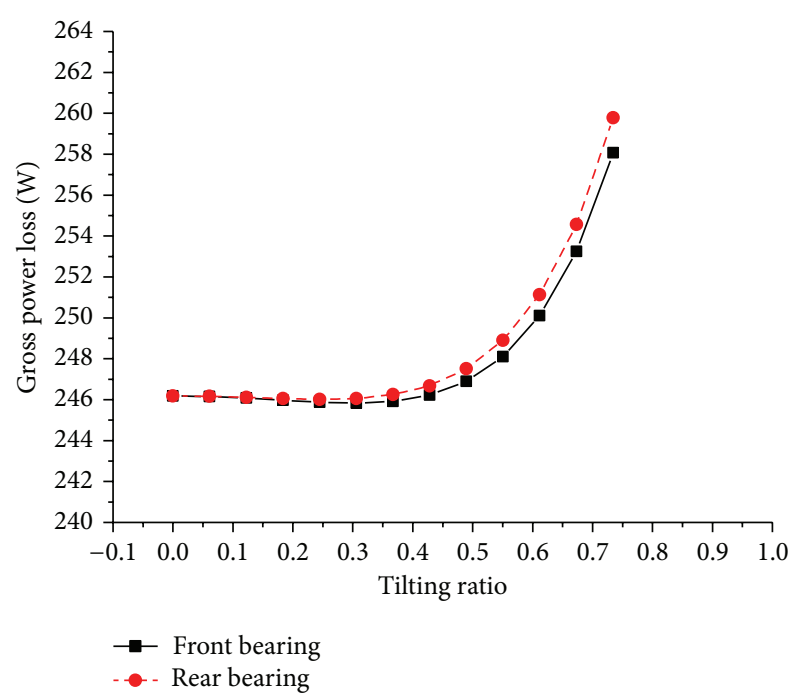

(b) The effect of tilting angle on the power loss

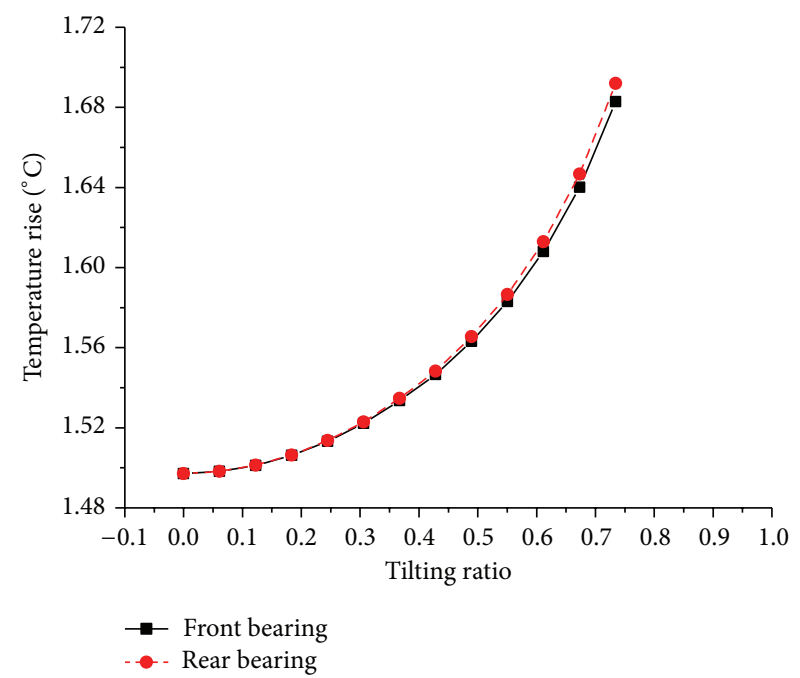

(c) The effect of tilting angle on the temperature rise

FIGURE 5: The effect of tilting angle on the static performance of the water-lubricated bearing. 


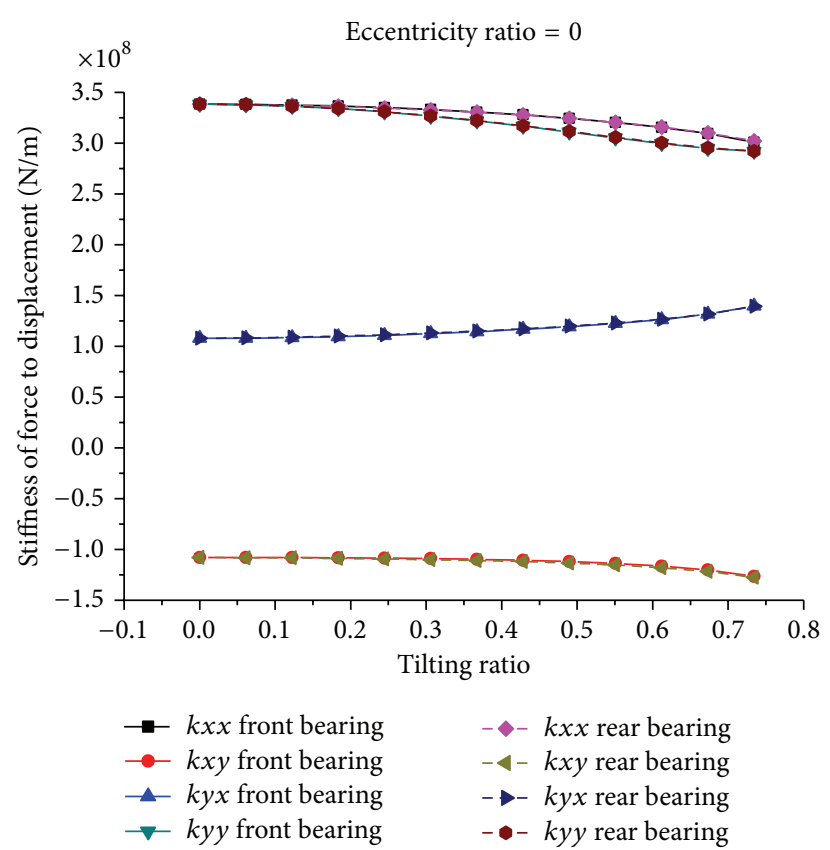

(a) The stiffness of force to displacement versus tilting ratio

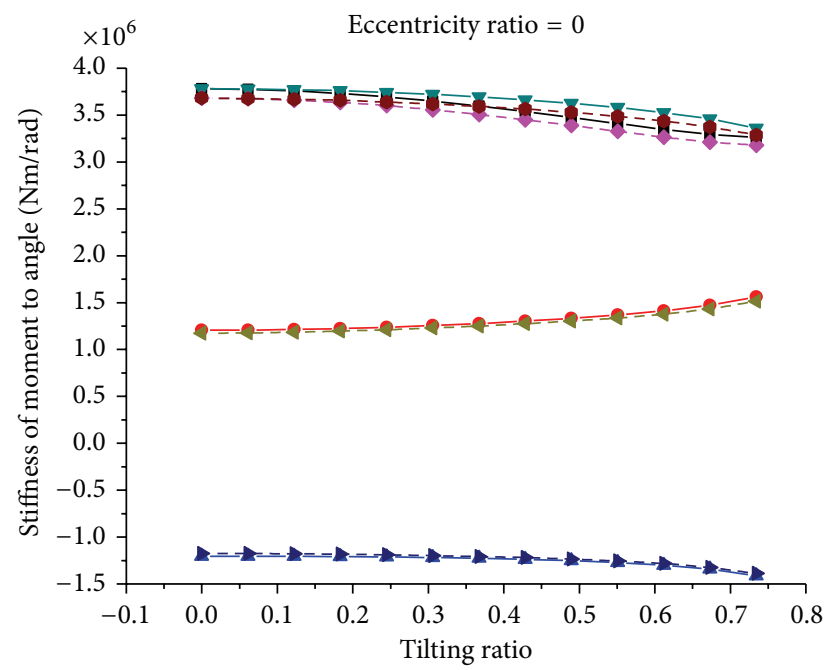

$\begin{array}{ll}-k m x \theta x \text { front bearing } & --k m x \theta x \text { rear bearing } \\ --k m x \theta y \text { front bearing } & -\checkmark-k m x \theta y \text { rear bearing } \\ -k m y \theta x \text { front bearing } & -k-k m y \theta x \text { rear bearing } \\ --k m y \theta y \text { front bearing } & -k m y \theta y \text { rear bearing }\end{array}$

(c) The stiffness of moment to angle versus tilting ratio

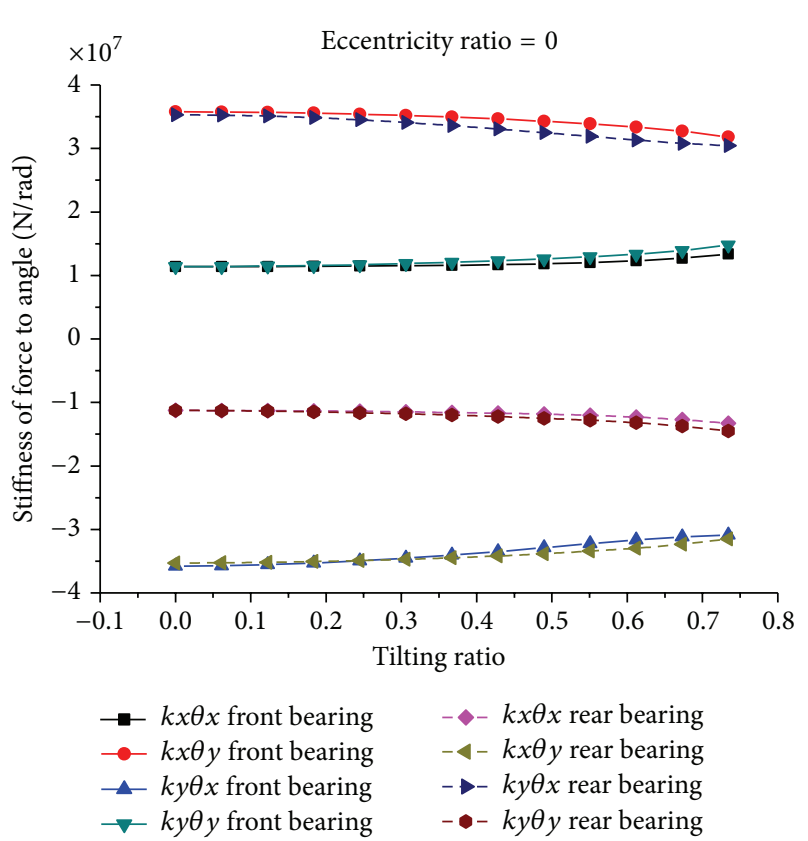

(b) The stiffness of force to angle versus tilting ratio

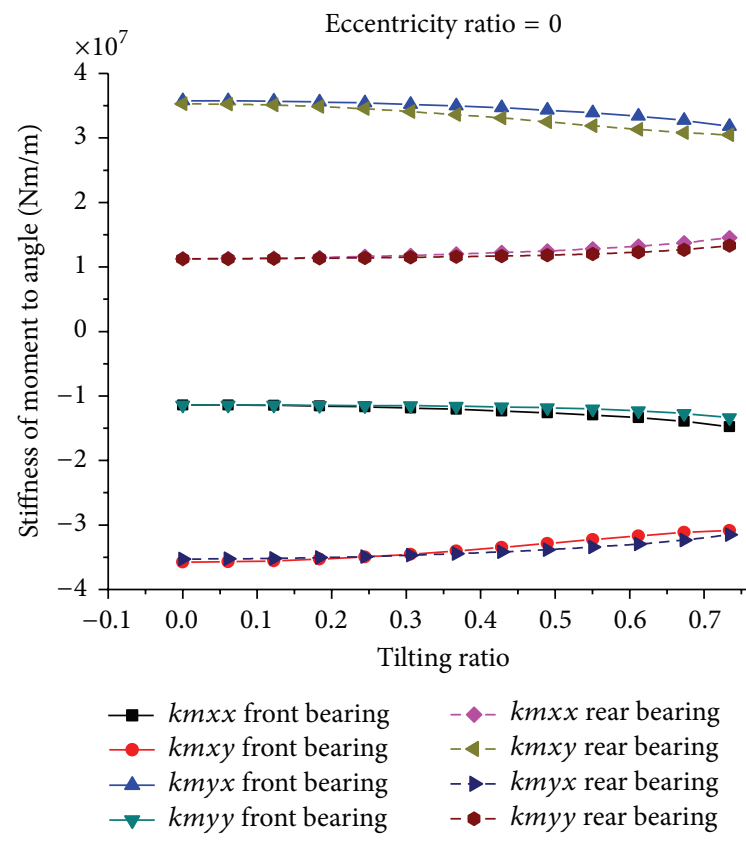

(d) The stiffness of moment to displacement versus tilting ratio

FIgURE 6: The effect of tilting ratio on the stiffness.

and validated with the experimental results available in the literature. A five-recess, turbulent-flow, water-lubricated hydrostatic bearing operating at a high rotational speed is tested by San Andres et al. [17]. Table 1 shows the bearing description and operating conditions and Figure 3 shows the schematic of the bearing. Figure 4 shows a comparison of measured load capacity and flow rate and the numerical predictions in respect to eccentricity ratio. As shown in Figure 4, a good agreement is observed between the present numerical predictions and the experimental results available in the reference. The calculated load capacity results correlate well with the experimental results, with a maximum difference of $11.5 \%$. The average of the flow rate for the experimental results is about $1.2 \mathrm{~kg} / \mathrm{s}$, while that for the numerical results is approximately $1.45 \mathrm{~kg} / \mathrm{s}$.

3.2. Effects of Tilting Ratio on the Static Performances of the Bearings. The geometric parameters for the bearings have been presented in Figure 1, the supply pressure is $1.5 \mathrm{MPa}$, the 

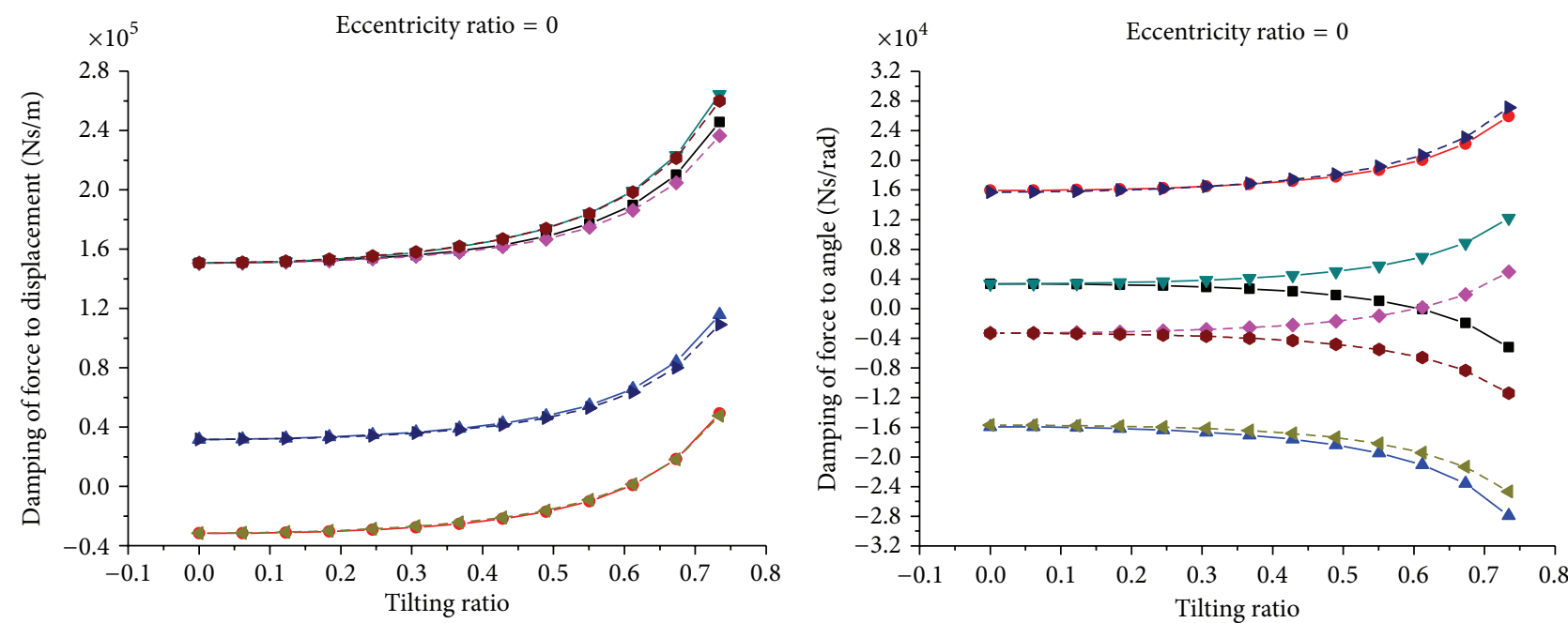

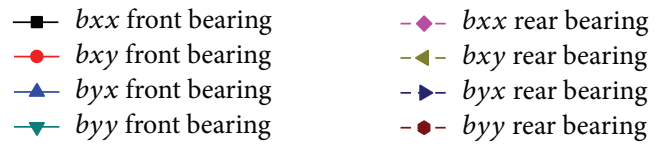

(a) The damping of force to displacement versus tilting ratio
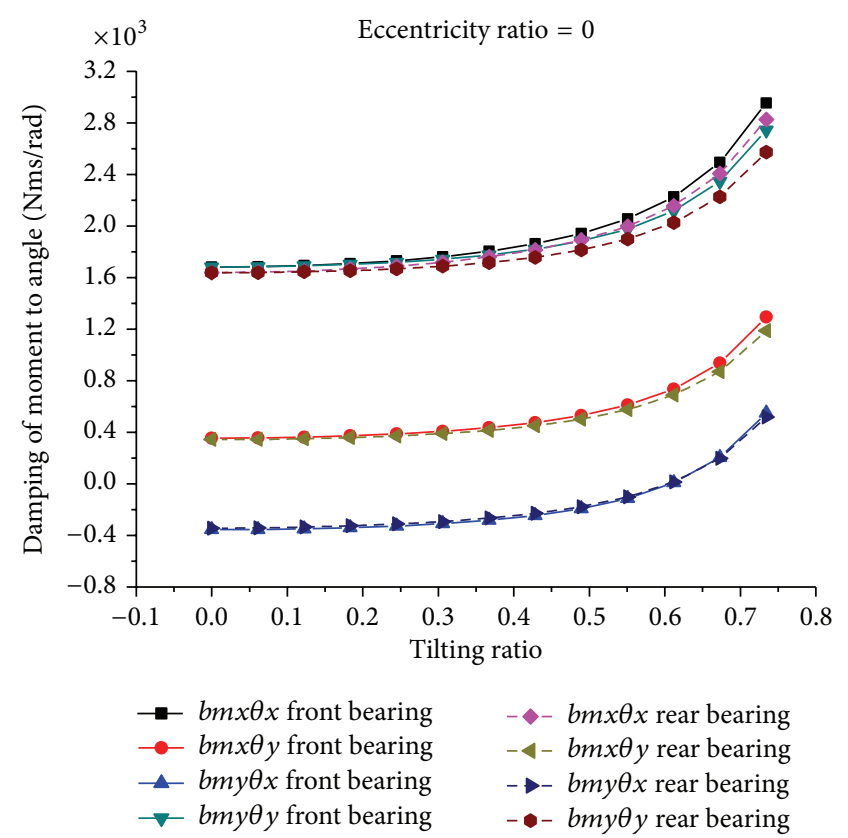

(c) The damping of moment to angle versus tilting ratio

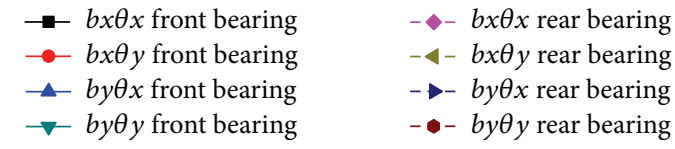

(b) The damping of force to angle versus tilting ratio

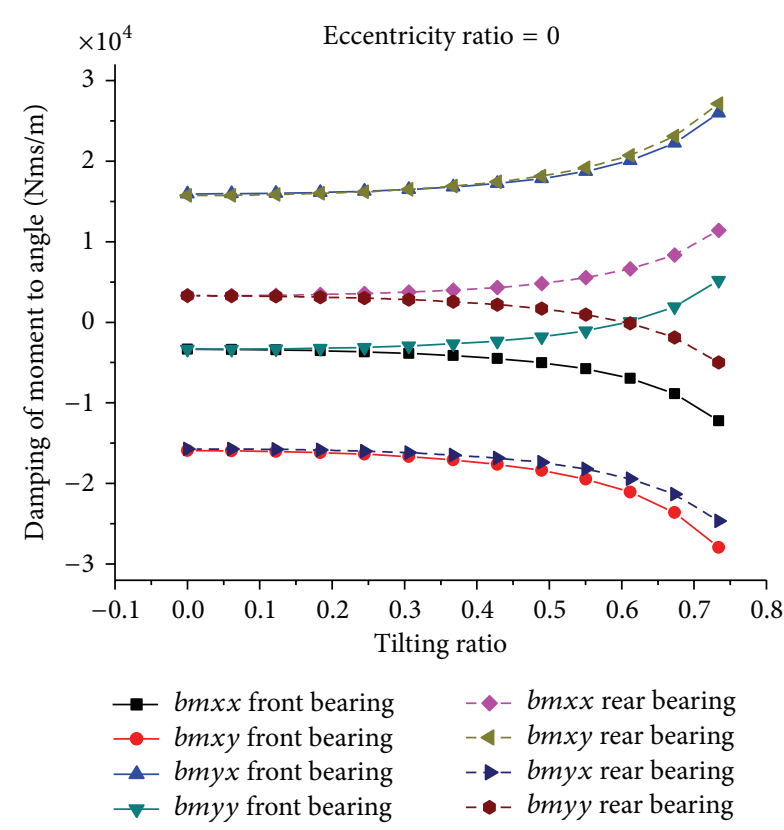

(d) The damping of moment to displacement versus tilting ratio

FIGURE 7: The effect of tilting ratio on the damping coefficients.

orifice diameter is $0.6 \mathrm{~mm}$, the pressurized water is supplied at a temperature of $30^{\circ} \mathrm{C}$, and the viscosity is $0.00087 \mathrm{~Pa} \cdot \mathrm{s}$.

Figure 1 shows the coordinate system for the tilting motion of the rotor. As the film variation of each bearing varies with different values for the tilting angles and bearing span, the tilting ratio is proposed here to investigate the effect of misalignment. The tilting ratio is defined as follows:

$$
\kappa=\frac{l_{m} \sqrt{\theta_{x}^{2}+\theta_{y}^{2}}}{c},
$$

where $l_{m}$ is the distance between the titling center and the left edge of the front bearing or the right edge of the rear bearing, which is shown in Figure 1.

In order to obtain a better physical insight into the effect of misalignment, the static characteristics have been presented with eccentricity ratio equal to zero. Figures 5(a)5(c) show the calculated quality, power loss, and temperature rise for different values of tilting ratios for each journal bearing operating at $10000 \mathrm{rpm}$. It is observed that the quality of each bearing undergoes a reduction by only $6.3 \%$ when 


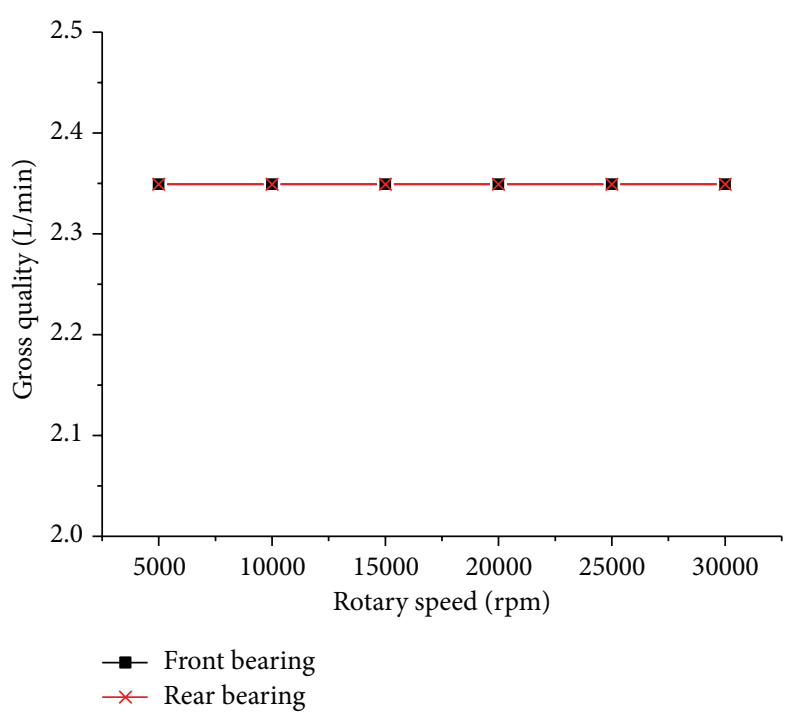

(a) The effect of rotary speed on the quality

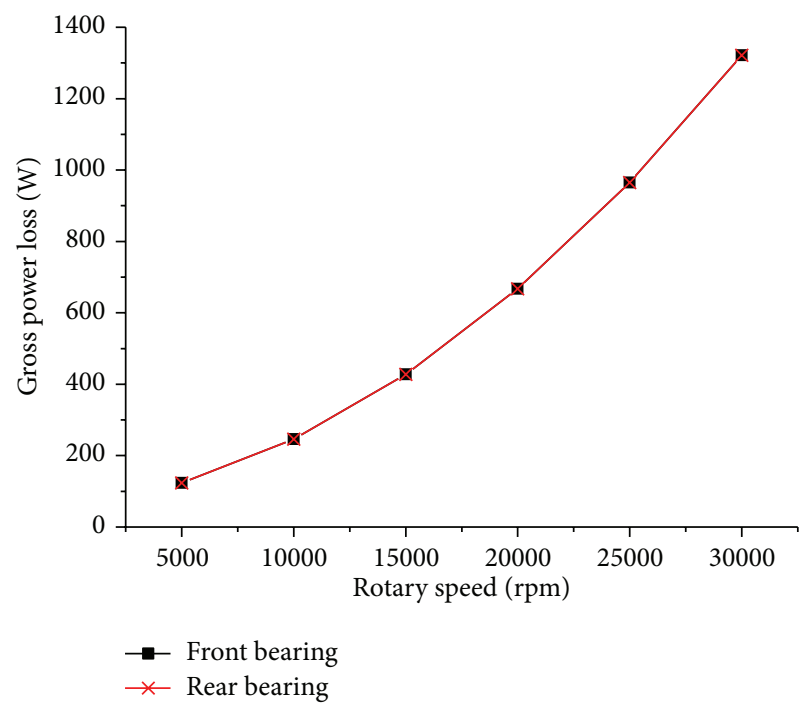

(b) The effect of rotary speed on the power loss

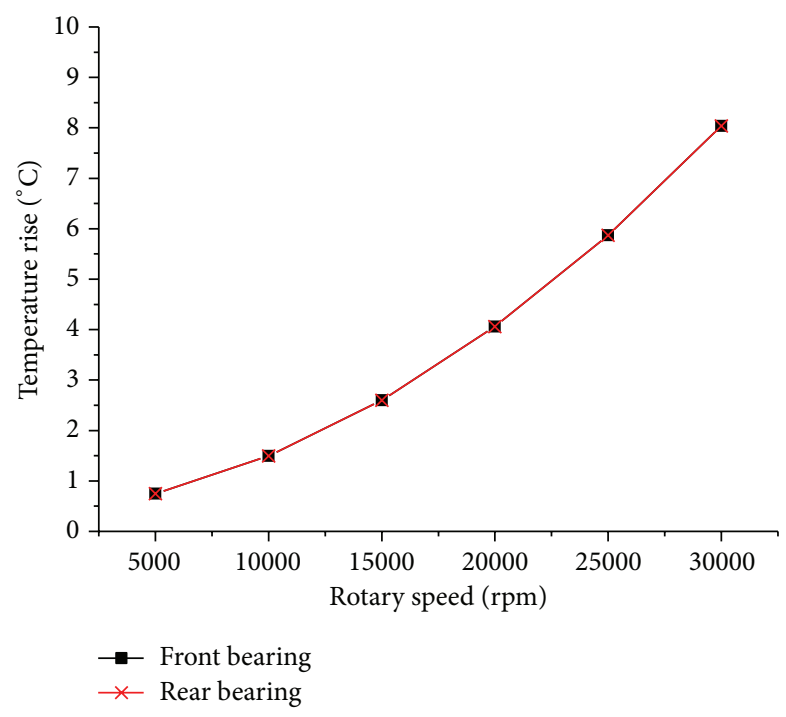

(c) The effect of rotary speed on the temperature rise

FIGURE 8: The effect of rotary speed on the static performance of the water-lubricated bearing.

the tilting ratio increases from zero to 0.75 . The total power loss of each bearing keeps almost unchanged when the tilting ratio is not greater than 0.4 . However, when the tilting ratio continues to increase, the power loss gets a slight increase by approximately 5\%. Meanwhile, the temperature rise increases with increased tilting ratio. The reason for this is that increasing tilting angles will increase the film variation of each bearing, which promote the hydrodynamic and turbulent effect. It is noticed that the maximum temperature rise for the investigated water-lubricated bearing is $1.7^{\circ} \mathrm{C}$ for the rotor-bearing system investigated here, which is far lower than the conventional oil film bearings. This in turn verifies the validation of the adiabatic assumption. In conclusion, when the tilting ratio is smaller than 0.4 , the influence of tilting ratio on the static performance of a water-lubricated hydrostatic journal bearing can be ignored.
3.3. Effects of Tilting Ratio on the Dynamic Characteristics of the Bearings. Figures 6 and 7 show the variation of the dynamic coefficients of the two identical bearings in respect to the tilting ratio with a rotary speed of $10000 \mathrm{rpm}$. The eccentricity ratio is assumed to be zero to exclude the effect of the clearance change due to the eccentricity ratio. The solid lines represent the dynamic coefficients of the front journal bearing while the dotted lines represent those of the rear bearing.

According to the results, the dynamic coefficients of force to displacement and moment to angle for the front bearing are approximately equal to those for the rear bearing due to the fact that the tilting center almost coincides with the bearing span center. However, the coupled dynamic coefficients of force to angle and moment to displacement for the two bearings have close proximity magnitudes but in the opposite 

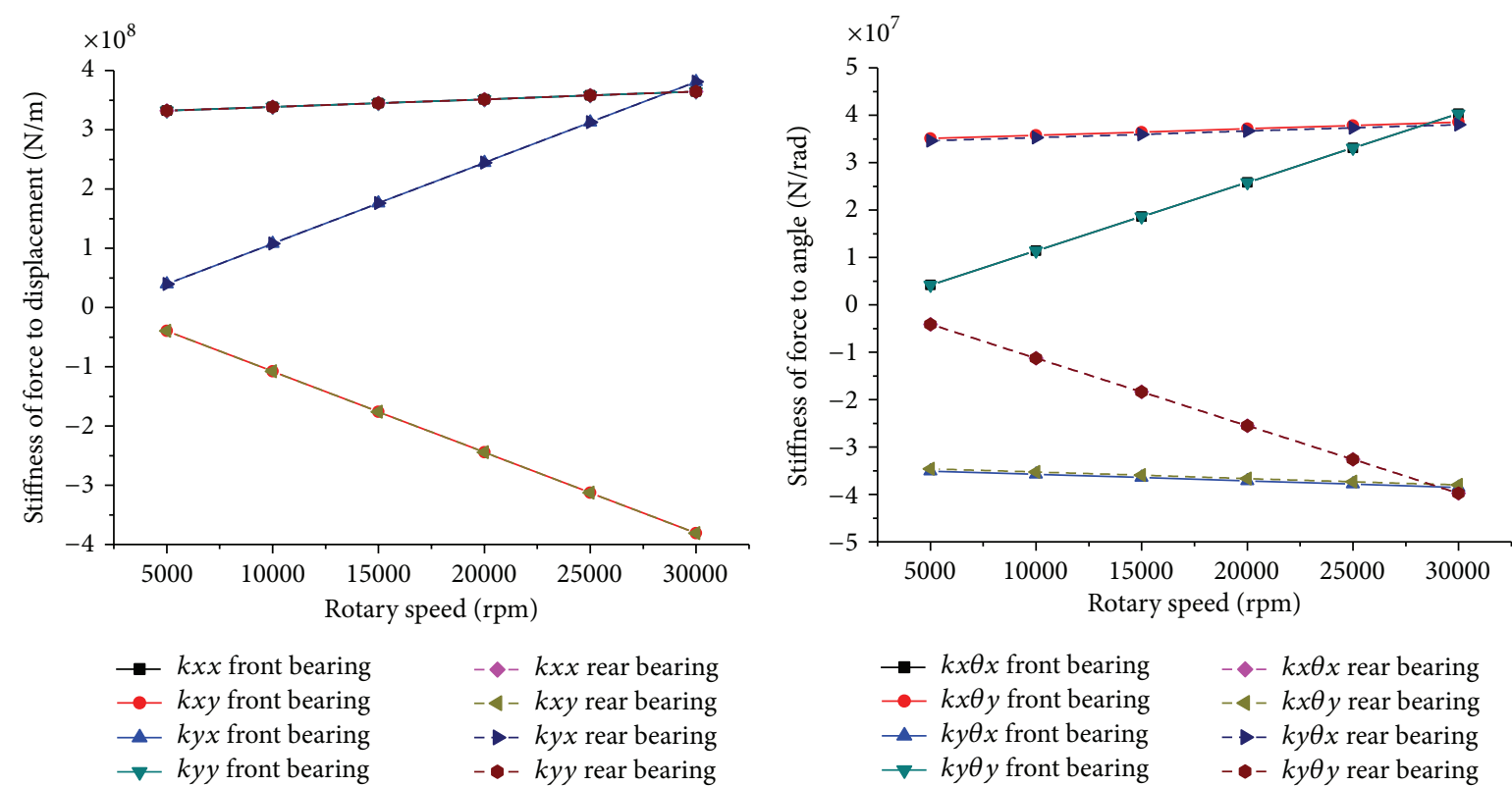

(a) The stiffness of force to displacement versys rotary speed
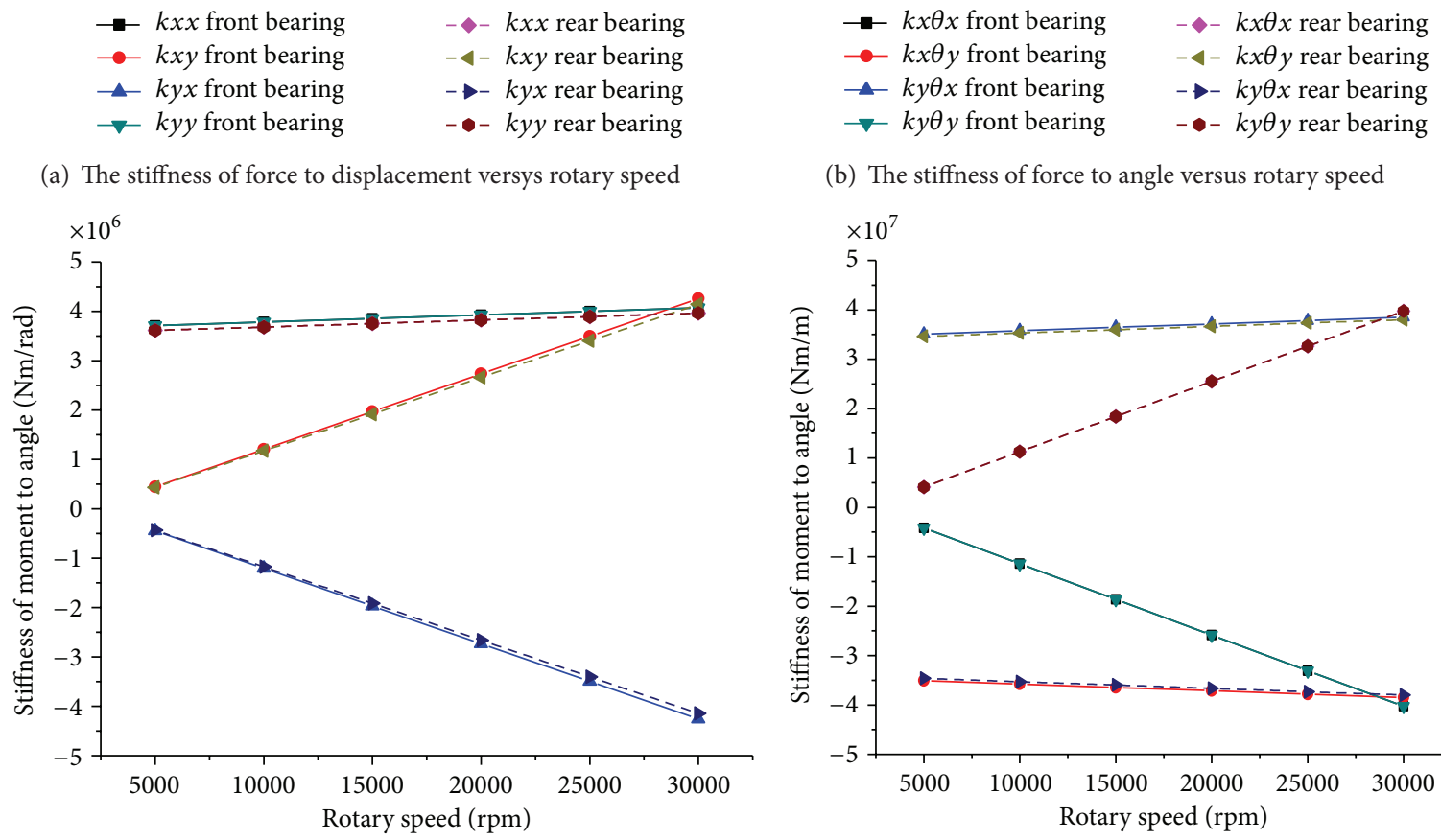

(b) The stiffness of force to angle versus rotary speed

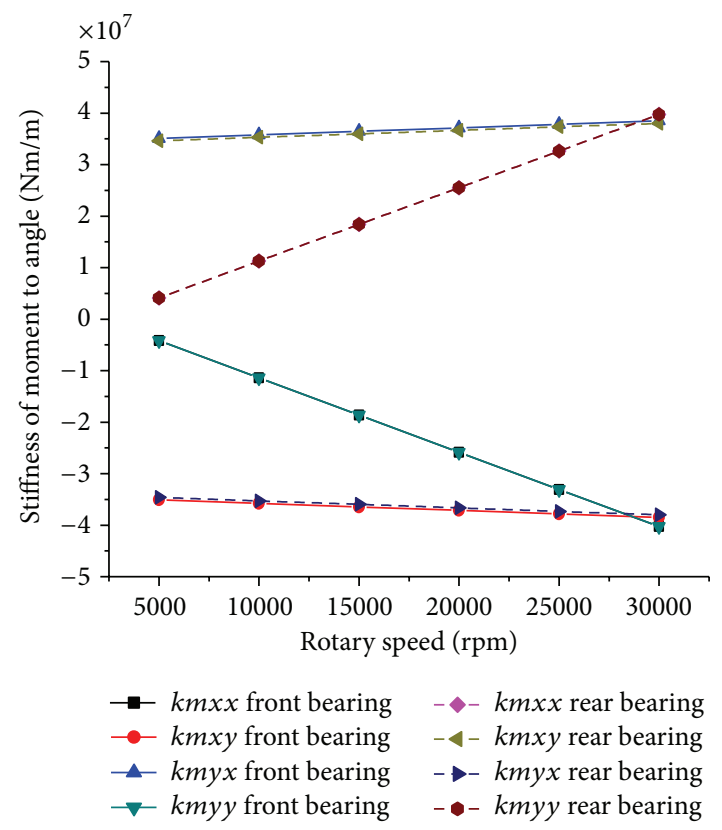

(c) The stiffness of moment to angle versus rotary speed

(d) The stiffness of moment to displacement versus rotary speed

FIgURE 9: The effect of rotary speed on the stiffness coefficients.

direction due to the fact that the two bearings are arranged at both sides of the mass center which is the origin of the tilting motions. It is observed that, with the increase of the tilting ratio, the stiffness and damping coefficients of the bearings keep almost independent of the tilting ratio when it is not larger than 0.4; however, when the tilting ratio continues to increase, the effect on dynamic coefficients is significant, with a maximum variation rate of $16.7 \%$ for the stiffness and $49.2 \%$ for the damping coefficients. Furthermore, the larger the tilting ratio is, the greater the differences among the coefficients are. This can be ascribed to the variations of the film thickness induced by the tilting angles and bearing span, which can be calculated according to (5). The results indicate that, for a small tilting ratio, the effect of the misalignment on the performance of hydrostatic waterlubricated journal bearings can be ignored; but for a relatively greater tilting ratio, the effect of misalignment should be taken into consideration.

3.4. Effects of Rotary Speed on the Static Performances of the Bearings. Figure 8 depicts the variation of the static characteristics for each journal bearing in respect to 

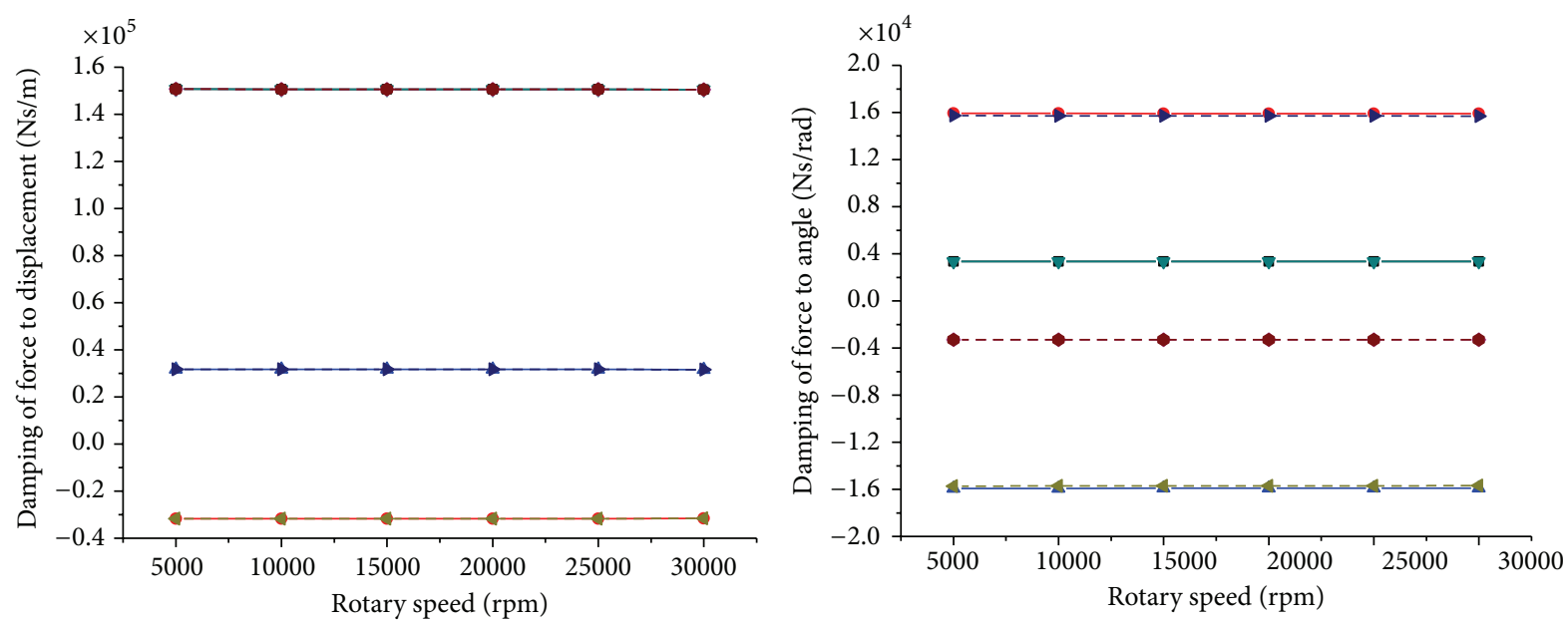

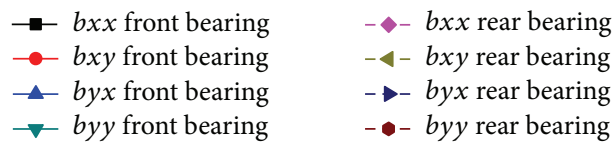

(a) The damping of force to displacement versus rotary speed

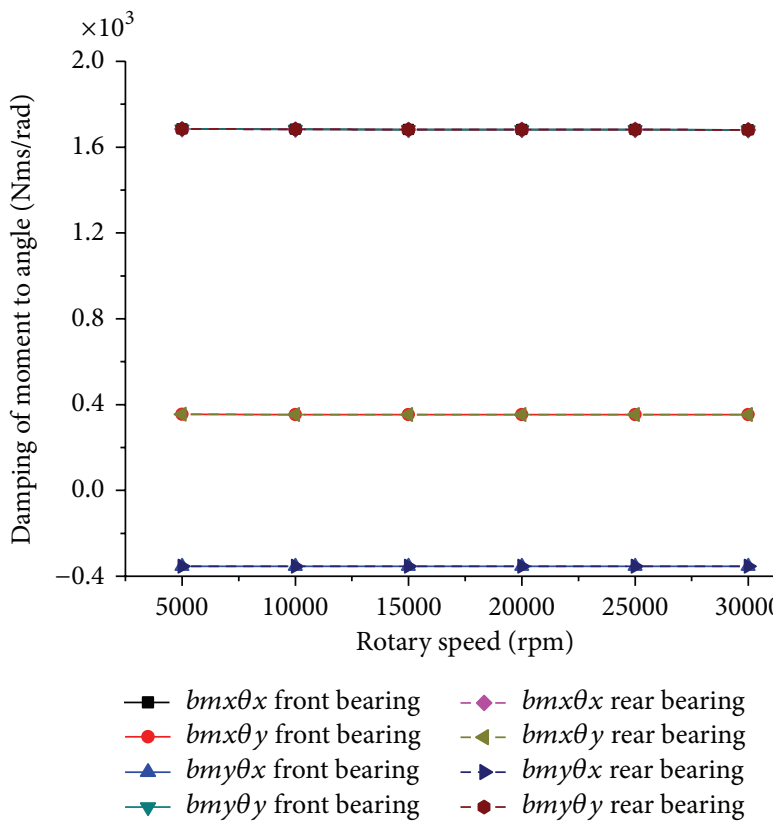

(c) The damping of moment to angle versus rotary speed $\rightarrow b x \theta x$ front bearing

- $b x \theta y$ front bearing

$\_b y \theta x$ front bearing

$\rightarrow$ by $\theta y$ front bearing

- $b x \theta x$ rear bearing

$-\triangleleft-b x \theta y$ rear bearing

$\rightarrow-b y \theta x$ rear bearing

- by $\theta y$ rear bearing

(b) The damping of force to angle versus rotary speed

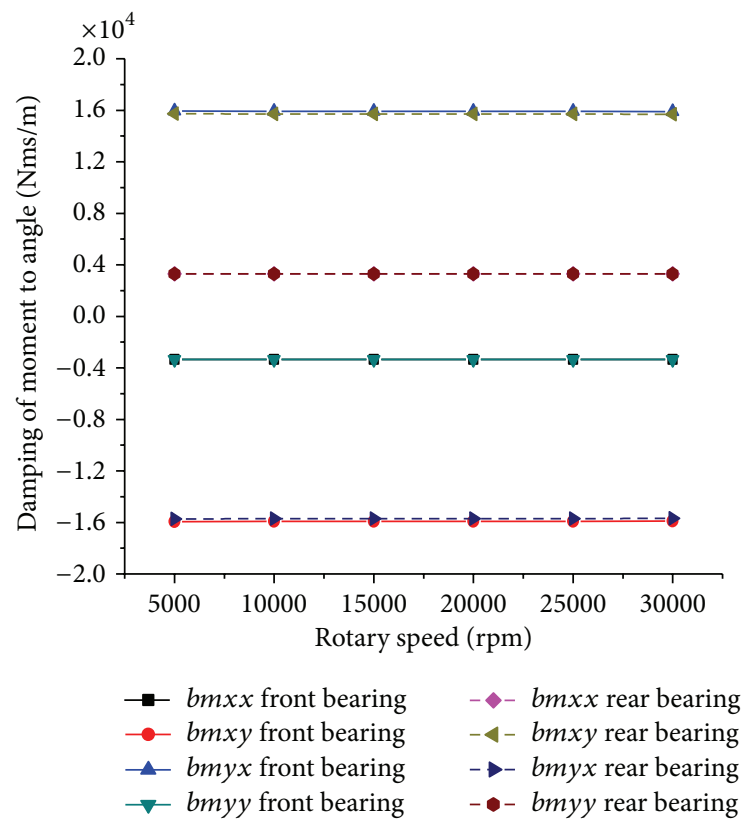

(d) The damping of moment to displacement versus rotary speed

Figure 10: The effect of rotary speed on the damping coefficients.

the rotary speed. In the case of a water-lubricated, hydrostatic bearing operating with eccentricity ratio and tilting angles equal to zero, it may be observed that the static characteristics of the front bearing are the same as those of the rear bearing. As shown in Figure 8(a), the quality of each bearing apparently remains unchanged with increased rotary speed. As shown in Figures 8(b) and 8(c), the gross power loss for each bearing increases dramatically from $123 \mathrm{~W}$ to $1232 \mathrm{~W}$ when the rotary speed increases from $5000 \mathrm{rpm}$ to $30000 \mathrm{rpm}$ because the frictional power loss is closely related to the rotary speed according to (12). Furthermore, the temperature rise undergoes a sharp increase from $0.7^{\circ} \mathrm{C}$ to $8^{\circ} \mathrm{C}$. It should be pointed out that most of the predicted temperature rises are higher than the actual values presumably due to the adiabatic assumption imposed on the analysis. Considering the values of the temperature rise, the adiabatic flow assumption is fully justified for the bearing studied. However, when the rotary speed continues to increase, the energy equation should be included to predict the temperature rise precisely.

3.5. Effects of Rotary Speed on the Dynamic Characteristics of the Bearings. Figures 9 and 10 show the dynamic coefficients 


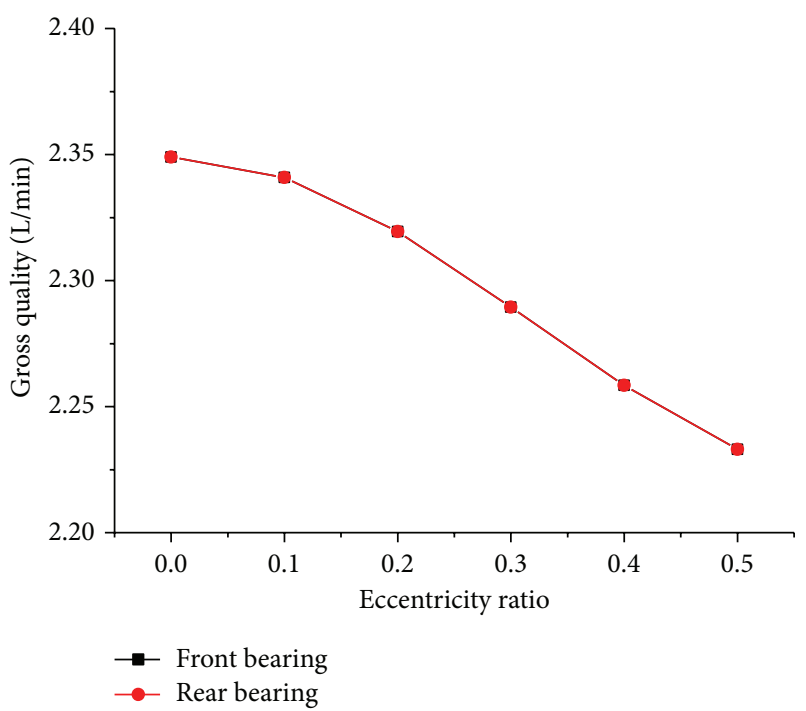

(a) The effect of rotary speed on the quality

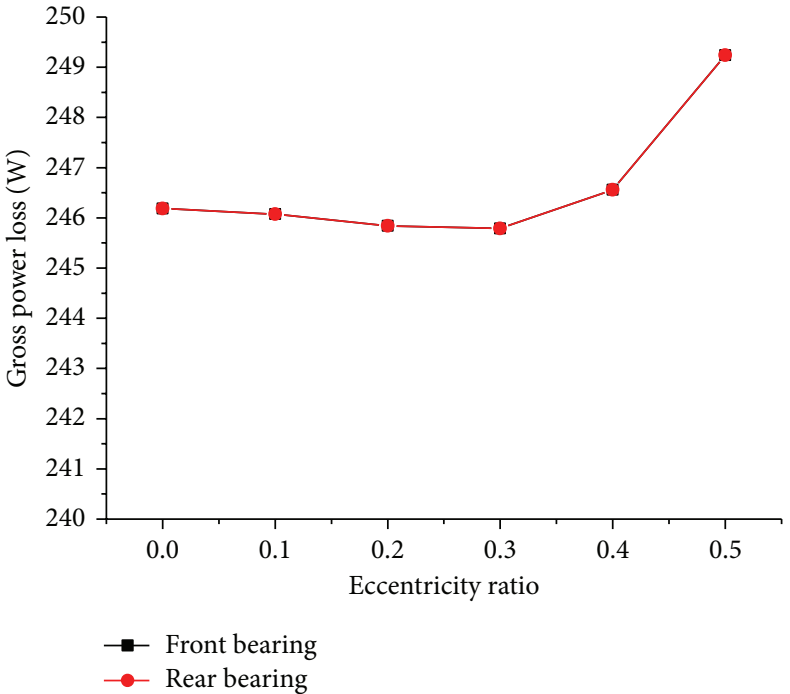

(b) The effect of rotary speed on the power loss

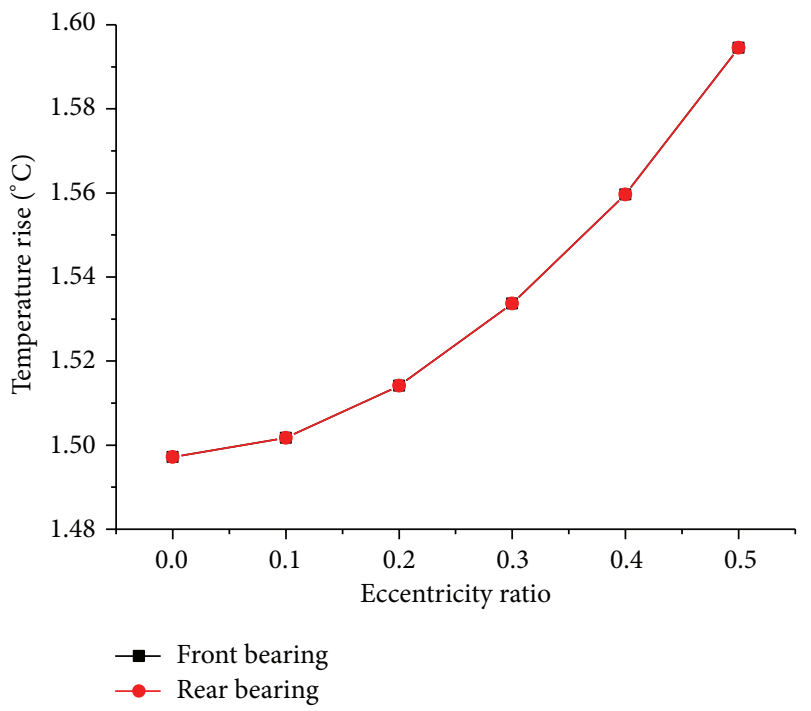

(c) The effect of rotary speed on the temperature rise

FIGURE 11: The effect of eccentricity ratio on the static performance of the water-lubricated bearing.

of each water-lubricated bearing in respect to the rotary speed. The eccentricity ratio and tilting angles are assumed as zero to exclude their influences on the film thickness. According to the results, a higher rotary speed generates larger coupled stiffness of force to displacement and moment to angle, but the influence of rotary speed on the relative direct coefficients is small. The direct stiffness coefficients of force to angle and moment to displacement are relatively insensitive to the variation of the rotary speed. The magnitudes of the cross-coupled stiffness of force to displacement and moment to angle are comparable to those of the direct stiffness, which demonstrates the importance of hydrodynamic effects. Unlike the stiffness, the damping coefficients are independent of the rotary speed in the aligned condition. The reason for this is that the perturbed pressure due to the perturbed velocity is not related to the rotary speed of the rotor.
3.6. Effects of Eccentricity Ratio on the Static Performances of the Bearings. The influence of eccentricity ratio in aligned condition on static performances of each bearing is as shown in Figure 11. The tilting ratio is assumed as zero to exclude the influence of tilting effect. Figure 11(a) indicates that the value of quality for each bearing decreases slowly with the eccentricity ratio. As is shown in Figure 11(b), the power loss is almost constant at first and then increases with increased eccentricity ratio. The maximum temperature rise across the bearing length is about $1.6^{\circ} \mathrm{C}$ at the eccentricity ratio equal to 0.5 . This is expected since a smaller clearance produces a larger frictional power loss and a smaller flow rate. However, it should be pointed out that in the aligned condition the static characteristics of the hydrostatic waterlubricated journal bearings vary slightly with eccentricity ratio. 


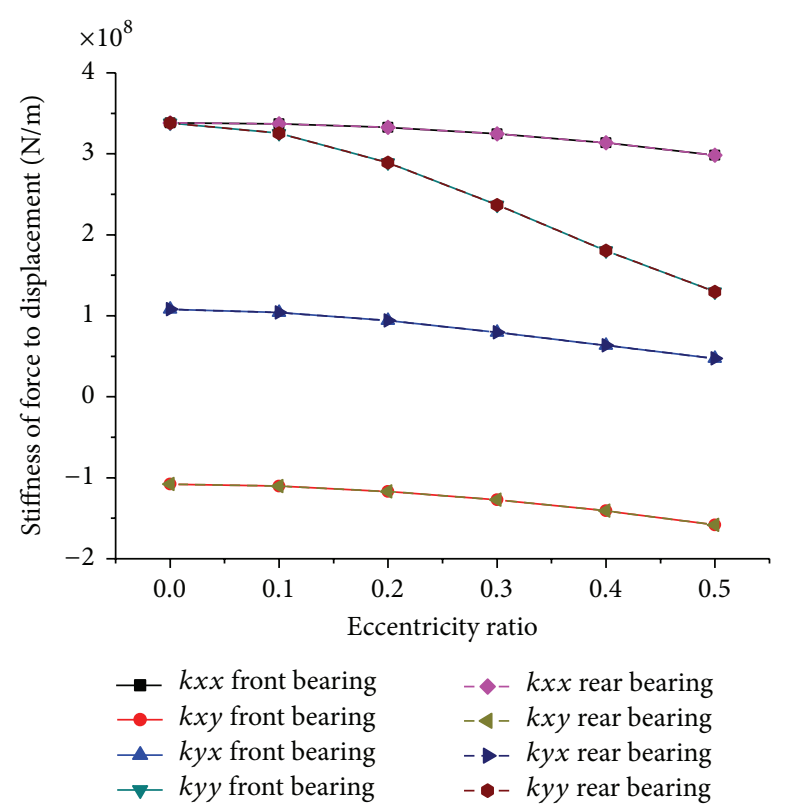

(a) The stiffness of force to displacement versus eccentricity ratio

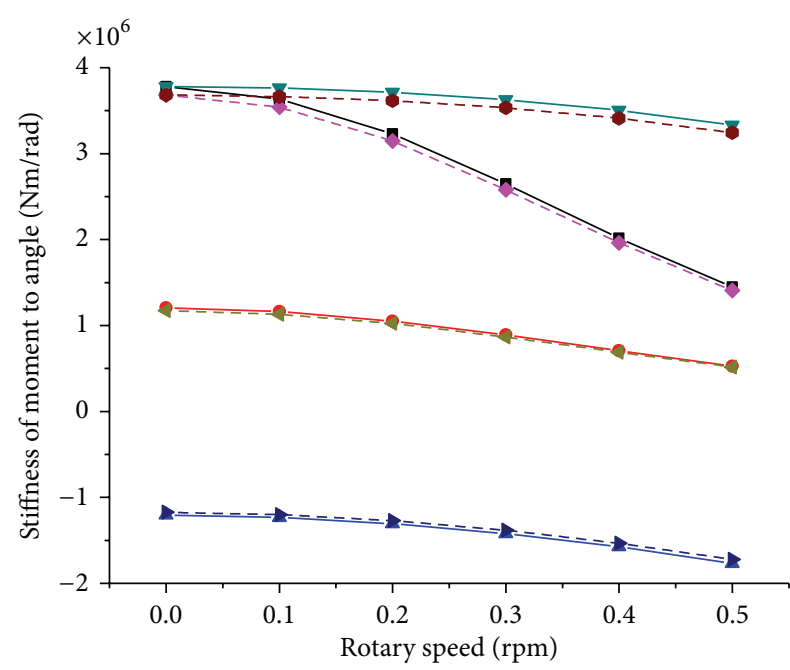

(c) The stiffness of moment to angle versus eccentricity ratio
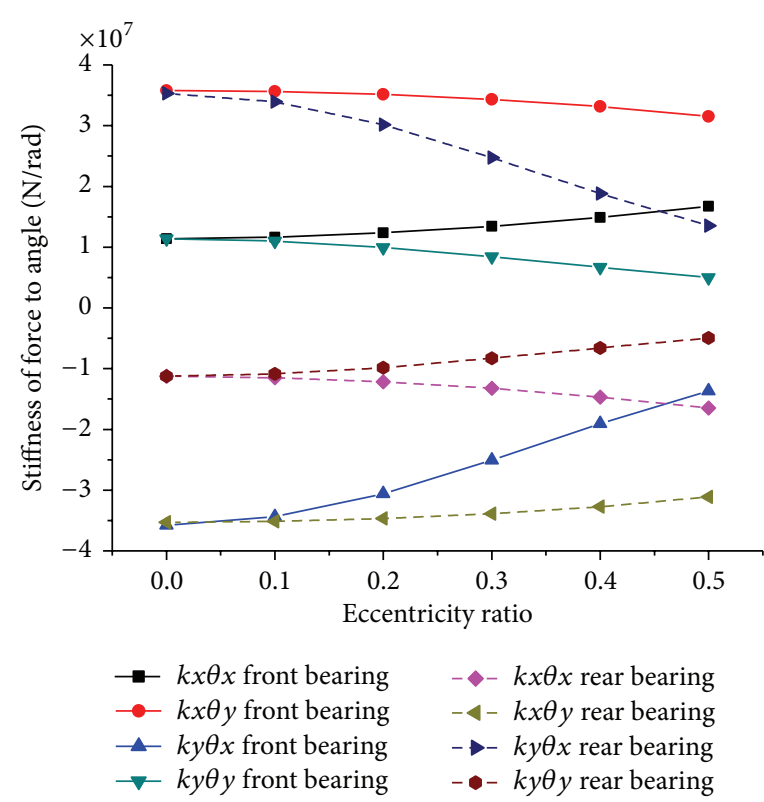

(b) The stiffness of force to angle versus eccentricity ratio

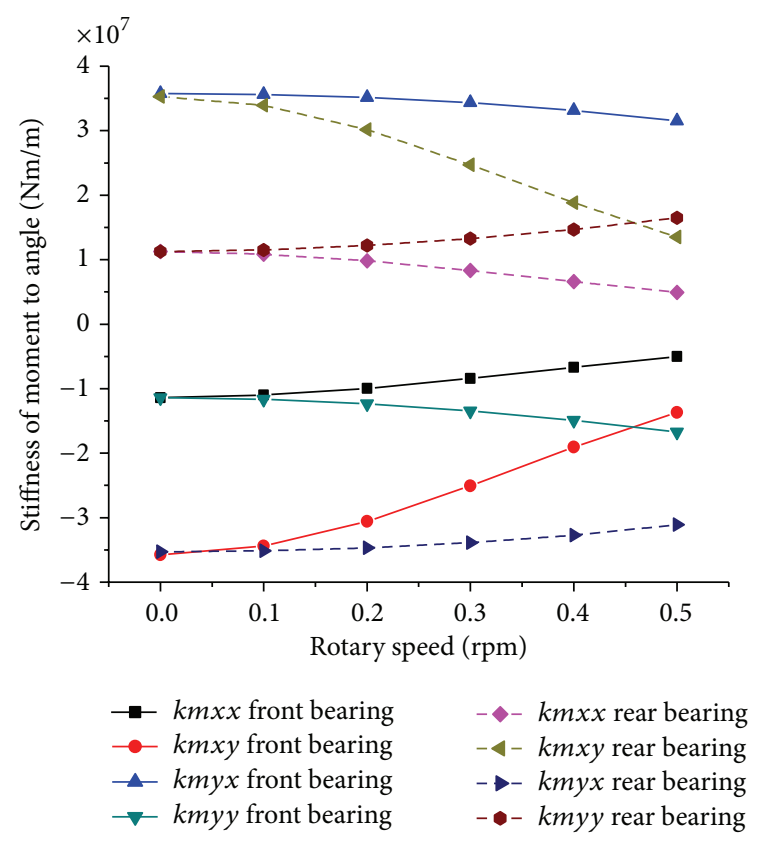

(d) The stiffness of moment to displacement versus eccentricity ratio

FIGURE 12: The effect of eccentricity ratio on the stiffness coefficients.

\subsection{Effects of Eccentricity Ratio on the Dynamic Characteristics} of the Bearings. Figures 12 and 13 show a variation of stiffness and damping coefficients of each bearing versus the eccentricity ratio. It may be noticed that the dynamic coefficients are almost constant as the eccentricity ratio increases from 0 to 0.1 . The direct stiffness of force to displacement and moment to angle decreases gradually with increased eccentricity ratio. Generally, the coupled stiffness varies significantly with the eccentricity ratio. The coupled coefficients of force to angle and moment to displacement for the front bearing have the same magnitude as those for the rear bearing, but in the opposite direction. The damping coefficients are also relatively insensitive to the eccentricity ratio when it is not larger than 0.1 . However, in larger eccentric condition, the damping coefficients vary with eccentricity ratio, and the larger the eccentricity ratio is, the greater the coefficients change. In summary, for the small eccentric condition $(\leq 0.1)$, the influence of eccentricity ratio on the full dynamic coefficients for the hydrostatic, waterlubricated journal bearing operating in aligned condition can 


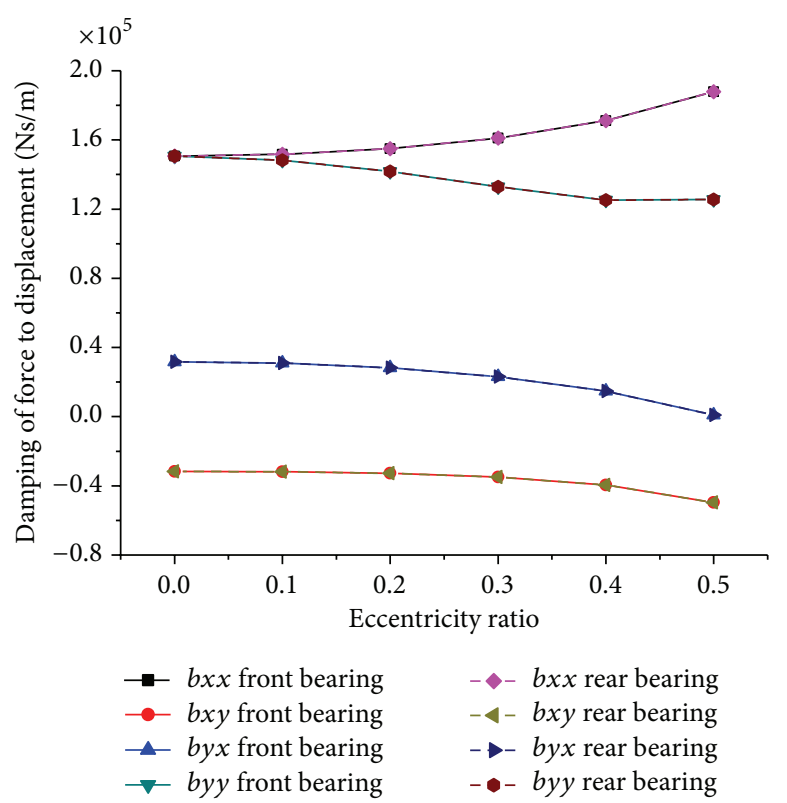

(a) The damping of force to displacement versus eccentricity ratio

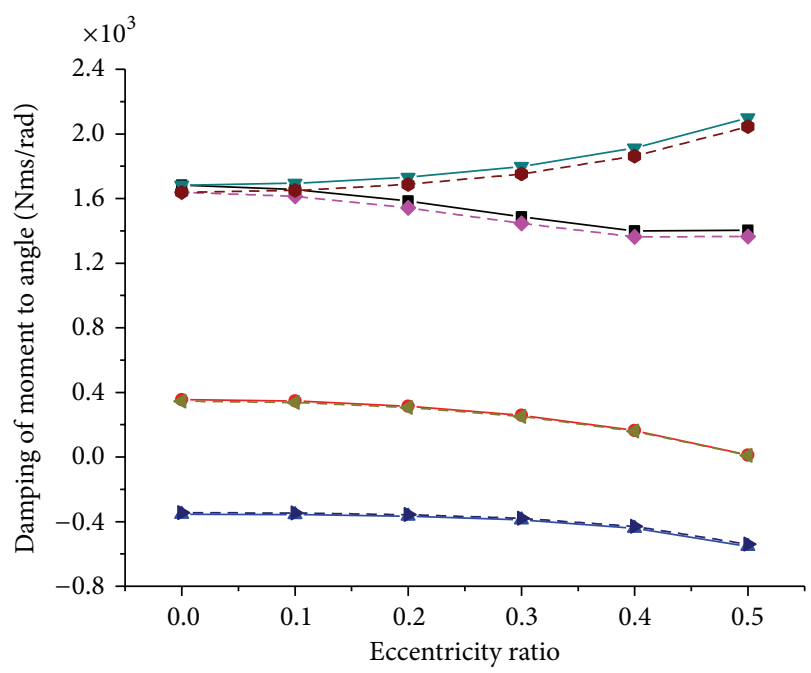

$\rightarrow b m x \theta x$ front bearing $\quad-\quad b m x \theta x$ rear bearing $\rightarrow b m x \theta y$ front bearing

— $b m y \theta x$ front bearing

$\nabla b m y \theta y$ front bearing

$-b m x \theta x$ rear bearing

$\rightarrow-b m y \theta x$ rear bearing

- bmy $\theta y$ rear bearing

(c) The damping of moment to angle versus eccentricity ratio

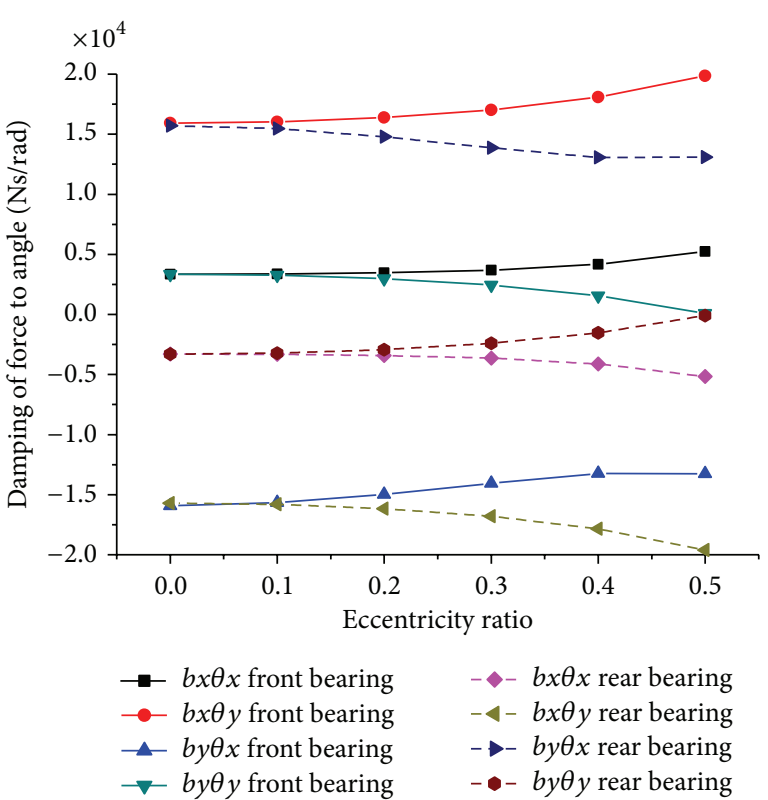

(b) The damping of force to angle versus eccentricity ratio

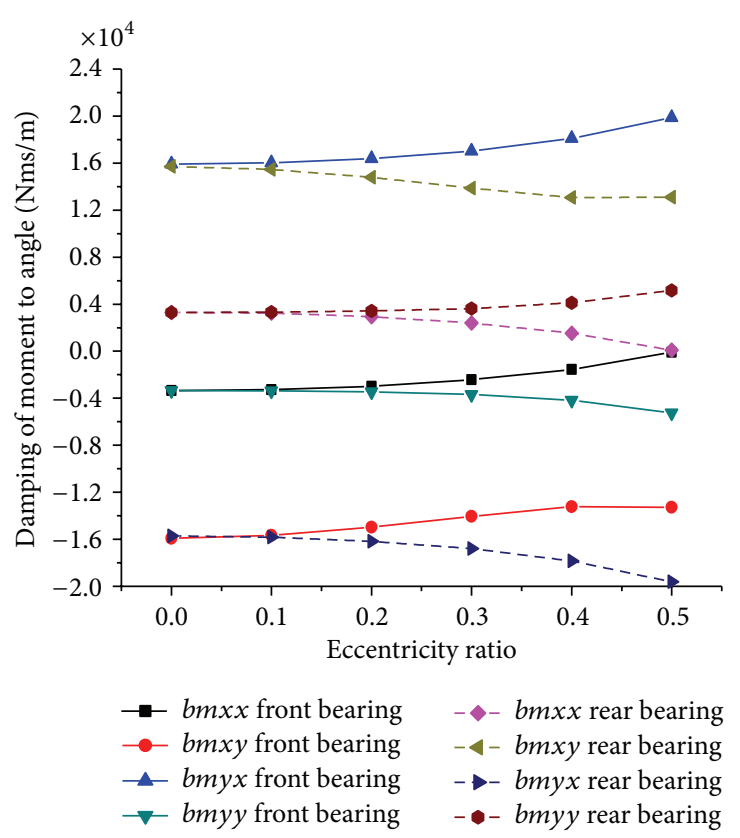

(d) The damping of moment to displacement versus eccentricity ratio

FIGURE 13: The effect of eccentricity ratio on the damping coefficients.

be ignored; however, for a larger eccentric condition, the influence should be fully discussed.

\section{Conclusion}

This paper investigated the complete dynamic coefficients for two hydrostatic, water-lubricated journal bearings used to support a rigid rotor considering the translational and tilting motion. The bulk turbulent flow model and FDM method is used to numerically predict the performance of the bearings. The results show that the proposed water-lubricated hydrostatic bearing has the potential to fulfill the target of lower power loss, temperature rise, and larger load capacity at high speed. On the basis of the results presented, the following conclusions can be drawn.

(1) For a small tilting ratio $(<0.4)$, the influence of tilting ratio on the static and dynamic characteristics of a water-lubricated hydrostatic journal bearing is relatively small; however, when the tilting ratio continues 
to increase, the power loss and temperature rise increase gradually while the quality decreases, and the effect of tilting ratio on the dynamic coefficients should be taken into consideration.

(2) The quality of the bearings is relatively insensitive to the rotary speed; however, the power loss and temperature rise increase sharply with the rotary speed in an aligned condition. The direct stiffness coefficients vary significantly with the rotary speed due to the hydrodynamic effect while the damping coefficients are almost constant.

(3) For a relatively smaller eccentric condition $(\leq 0.1)$, the static and dynamic characteristics of the hydrostatic water-lubricated journal bearings vary slightly with eccentricity ratio. However, for a larger eccentric condition, the dynamic characteristics increase or decrease significantly with the eccentricity ratio.

\section{Nomenclature}

$c$ : Design film thickness

$d_{0}$ : Orifice diameter

$h$ : Film thickness

$l_{1}$ : Distance between the mass center and front journal bearing center

$l_{2}$ : Distance between the mass center and rear journal bearing center

$l_{m 1}$ : Distance between the mass center and the left edge of front journal bearing

$l_{m 2}$ : Distance between the mass center and the right edge of rear journal bearing

$r$ : Radius of the bearing

$D$ : Diameter of a journal bearing

$F$ : The bearing force

$L$ : The length of a journal bearing

$P_{s}$ : Supply pressure

$W$ : The external load

$\Omega:$ Rotary speed

$\alpha$ : Flow coefficient

$\rho$ : Density

$\mu: \quad$ Viscosity

$\lambda$ : Orifice design coefficient.

\section{Conflict of Interests}

The authors declare that there is no conflict of interests regarding the publication of this paper.

\section{References}

[1] F. Liu, B. Lin, and X. F. Zhang, "Numerical design method for water-lubricated hybrid sliding bearings," International Journal of Precision Engineering and Manufacturing, vol. 9, no. 1, pp. 4750, 2008.

[2] X. Y. Yuan, G. Y. Zhang, B. Li, and X. Miao, "Theoretical and experimental results of water-lubricated, high-speed, shortcapillary-compensated hybrid journal bearings," in Proceedings of IJTC, STLE/ASME International Joint Tribology Conference, pp. 391-398, San Antonio, Tex, USA, October 2006.
[3] S. Yoshimoto, T. Kume, and T. Shitara, "Axial load capacity of water-lubricated hydrostatic conical bearings with spiral grooves for high speed spindles," Tribology International, vol. 31, no. 6, pp. 331-338, 1998.

[4] G. Y. Gao, Z. W. Yin, D. Jiang et al., "Numerical analysis of plain journal bearing under hydrodynamic lubrication by water," Tribology International, vol. 75, pp. 31-38, 2014.

[5] S. Nakano, T. Kishibe, T. Inoue, and H. Shiraiwa, "An advanced microturbine system with water-lubricated bearings," International Journal of Rotating Machinery, vol. 2009, Article ID 718107, 12 pages, 2009.

[6] H. H. Feng, "Dynamic characteristics of a rigid spindle supported by water-lubricated bearings," Applied Mechanics and Materials, vol. 401-403, pp. 121-124, 2013.

[7] J. Corbett, R. J. Almond, D. J. Stephenson, and Y. B. P. Kwan, "Porous ceramic water hydrostatic bearings for improved for accuracy performance," CIRP Annals: Manufacturing Technology, vol. 47, no. 1, pp. 467-470, 1998.

[8] O. Ebrat, Z. P. Mourelatos, N. Vlahopoulos, and K. Vaidyanathan, "Calculation of journal bearing dynamic characteristics including journal misalignment and bearing structural deformation," Tribology Transactions, vol. 47, no. 1, pp. 94-102, 2004.

[9] S. C. Jain, S. C. Sharma, and T. Nagaraju, "Misaligned journal effects in liquid hydrostatic non-recessed journal bearings," Wear, vol. 210, no. 1-2, pp. 67-75, 1997.

[10] S. C. Sharma, V. M. Phalle, and S. C. Jain, "Combined influence of wear and misalignment of journal on the performance analysis of three-lobe three-pocket hybrid journal bearing compensated with capillary restrictor," Journal of Tribology, vol. 134, no. 1, Article ID 011703, 2012.

[11] G. H. Jang and J. W. Yoon, "Dynamic characteristics of a coupled journal and thrust hydrodynamic bearing in a HDD spindle system due to its groove location," Microsystem Technologies, vol. 8, no. 4-5, pp. 261-270, 2002.

[12] G. H. Jang and S. H. Lee, "Determination of the dynamic coefficients of the coupled journal and thrust bearings by the perturbation method," Tribology Letters, vol. 22, no. 3, pp. 239246, 2006.

[13] H. W. Kim, G. H. Jang, and S. H. Lee, "Complete determination of the dynamic coefficients of coupled journal and thrust bearings considering five degrees of freedom for a general rotorbearing system," Microsystem Technologies, vol. 17, no. 5-7, pp. 749-759, 2011.

[14] G. G. Hirs, "A bulk-flow theory for turbulence in lubricant films," Journal of Lubrication Technology, vol. 95, no. 2, pp. 137146, 1973.

[15] J. Frêne, M. Arghir, and V. Constantinescu, "Combined thinfilm and Navier-Stokes analysis in high Reynolds number lubrication," Tribology International, vol. 39, no. 8, pp. 734-747, 2006.

[16] R. Bassani, E. Ciulli, B. Piccigallo, M. Pirozzi, and U. Staffilano, "Hydrostatic lubrication with cryogenic fluids," Tribology International, vol. 39, no. 8, pp. 827-832, 2006.

[17] L. San Andres, D. Childs, and Z. Yang, "Turbulent-flow hydrostatic bearings: analysis and experimental results," International Journal of Mechanical Sciences, vol. 37, no. 8, pp. 815-829, 1995.

[18] M. M. Khonsari and E. R. Booser, Applied Tribology, WileyInterscience, London, UK, 2008. 


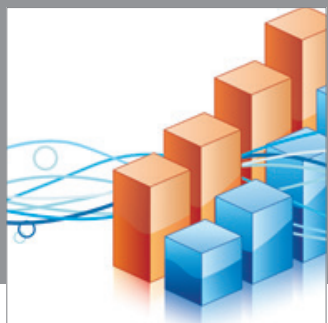

Advances in

Operations Research

mansans

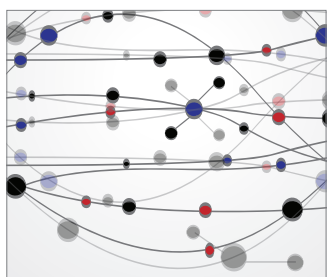

The Scientific World Journal
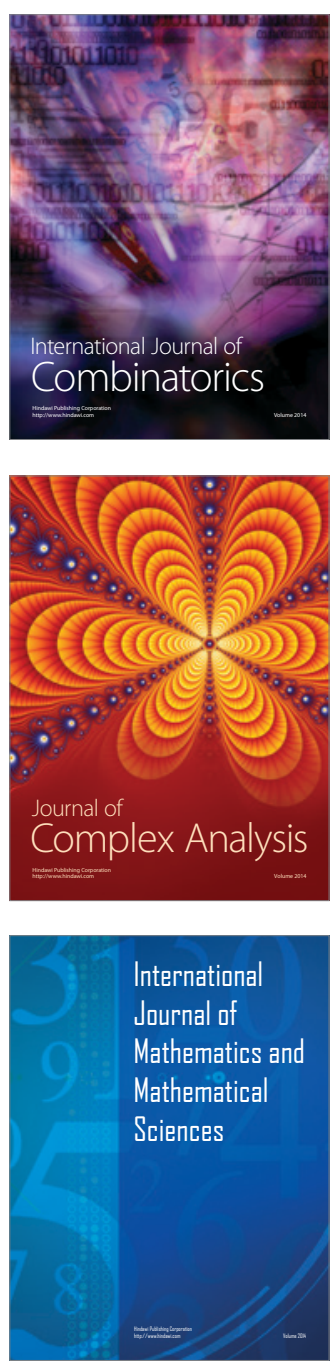
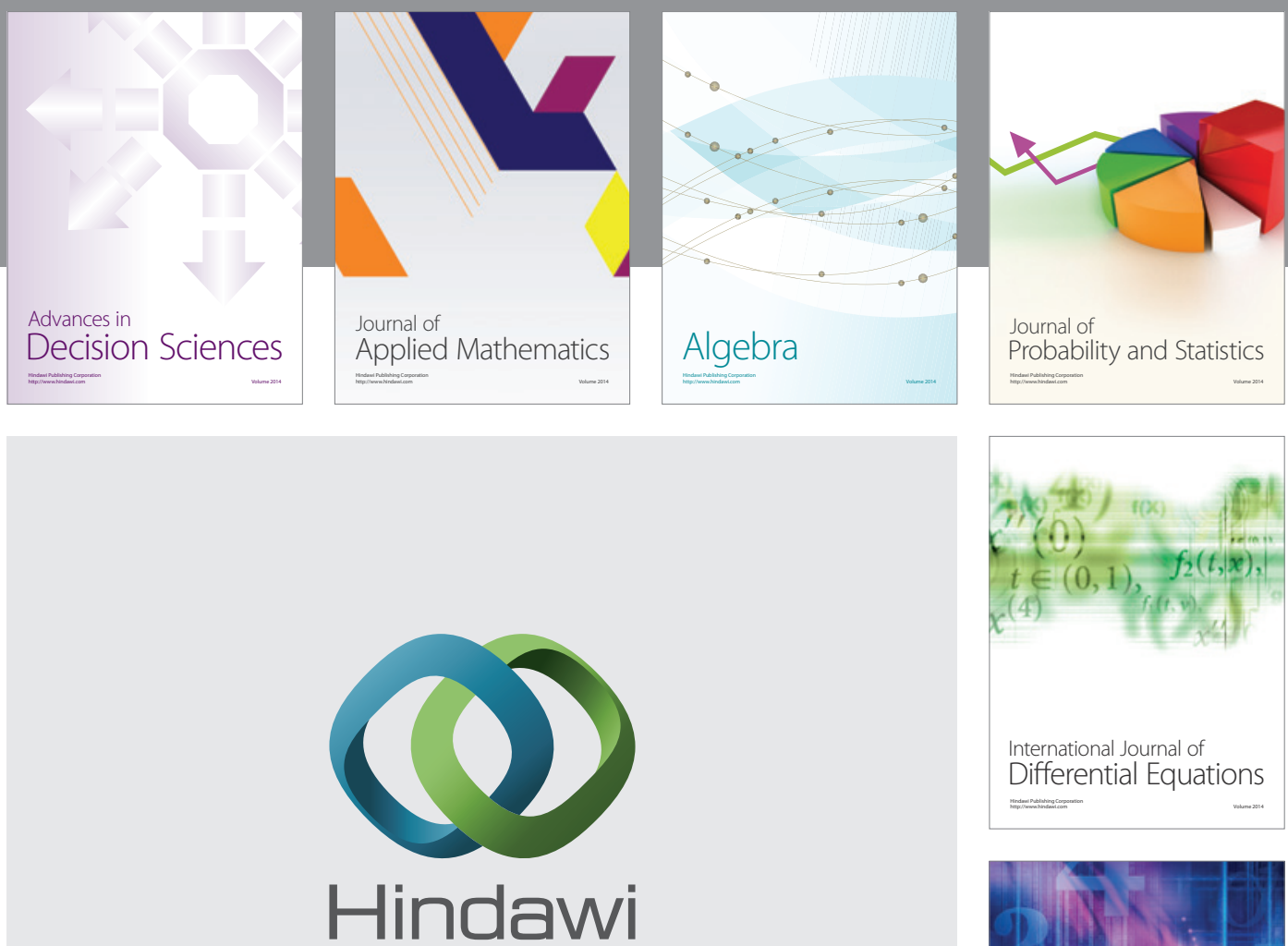

Submit your manuscripts at http://www.hindawi.com
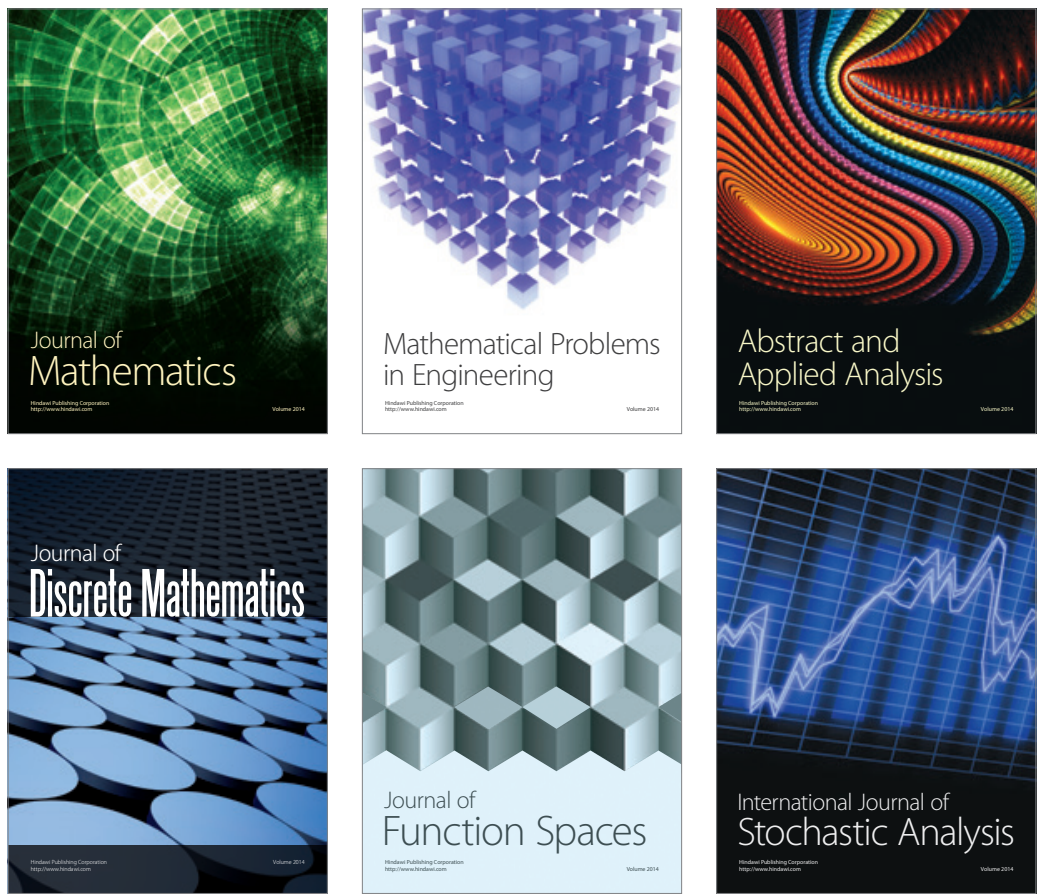

Journal of

Function Spaces

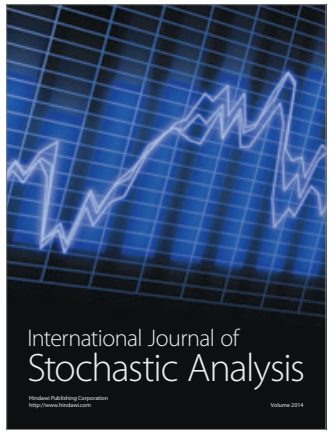

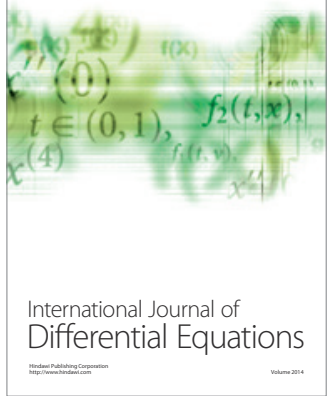
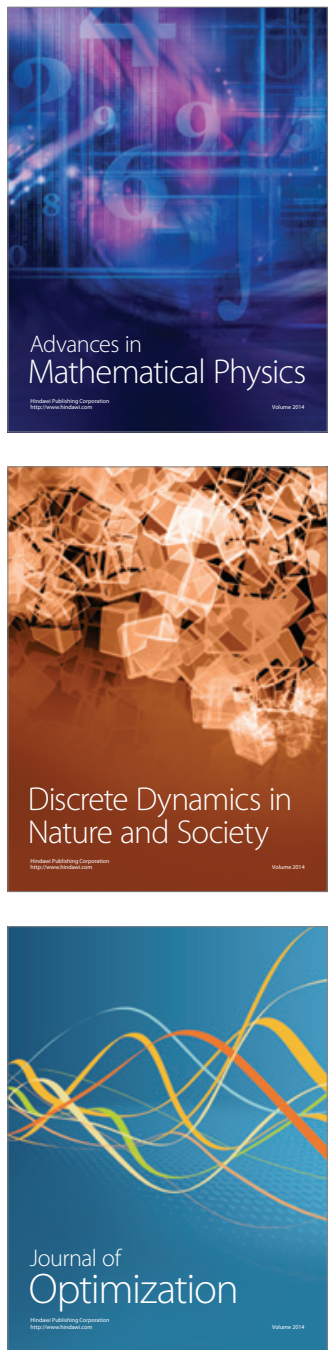